\title{
Analyse de l'evolution de la pratique de l'excision au Burkina Faso: L'environment institutionel, politique et programmatique de la lutte contre la pratique de l'excision
}

\author{
Nafissatou J. Diop \\ Population Council \\ Zakari Congo \\ Population Council \\ Aina Ouedraogo \\ Alphosine Sawadogo \\ Lydia Saloucou
}

See next page for additional authors

Follow this and additional works at: https://knowledgecommons.popcouncil.org/departments_sbsr-rh

Part of the Community-Based Research Commons, Community Health and Preventive Medicine Commons, Gender and Sexuality Commons, International Public Health Commons, Obstetrics and Gynecology Commons, Public Health Education and Promotion Commons, and the Women's Health Commons

How does access to this work benefit you? Let us know!

\section{Recommended Citation}

Diop, Nafissatou J., Zakari Congo, Aina Ouedraogo, Alphosine Sawadogo, Lydia Saloucou, and Ida Tamini. 2006. "Analyse de l'evolution de la pratique de l'excision au Burkina Faso: L'environment institutionel, politique et programmatique de la lutte contre la pratique de l'excision." Ouagadougou: Population Council. 


\section{Authors}

Nafissatou J. Diop, Zakari Congo, Aina Ouedraogo, Alphosine Sawadogo, Lydia Saloucou, and Ida Tamini 


\section{ANALYSE DE L'EVOLUTION DE LA PRATIQUE DE L'EXCISION AU BURKINA FASO}

L'ENVIRONNEMENT INSTITUTIONEL POLITIQUE ET PROGRAMMATIQUE DE LA LUTTE CONTRE LA PRATIQUE DE L'EXCISION 


\title{
ANALYSE DE L'EVOLUTION DE LA PRATIQUE DE L'EXCISION AU BURKINA FASO
}

\author{
L'ENVIRONNEMENT INSTITUTIONEL, POLITIQUE ET \\ PROGRAMMATIQUE DE LA LUTTE CONTRE LA PRATIQUE DE \\ L'EXCISION
}

\author{
Nafissatou J. Diop* \\ Zakari Congo* \\ Aïna Ouédraogo** \\ Alphonsine Sawadogo** \\ Lydia Saloucou* \\ Ida Tamini** \\ * Population Council \\ ** SP/CNLPE
}

Mars 2006

This study was funded by the UNITED STATES AGENCY FOR INTERNATIONAL DEVELOPMENT (USAID) under the terms of Cooperative Agreement Number HRN-A-00-98-00012-00 and Population Council Subagreement Number AI05.01A. The opinions expressed herein are those of the authors and do not necessarily reflect the views of USAID. 


\section{Liste des abréviations}

\begin{tabular}{l:l} 
APJAD & Association pour la Promotion de la Jeunesse Africaine et le Développement \\
CNLPE & Comité National de Lutte contre la Pratique de l’Excision \\
DPASSN & Direction Provinciale de l'Action Sociale et de la Solidarité Nationale \\
EDS & Enquête Démographique et de Santé \\
IEC/CCC & Information, Education, Communication/ Communication pour le changement de \\
LSC & Laboratoire de Santé Communautaire \\
MASSN & Ministère de l'Action Sociale et de la Solidarité Nationale \\
MASSN & Ministère de l'Action Sociale et de la Solidarité Nationale \\
PEBC & Programme d'Education à Base Communautaire \\
SP/CNLPE & Secrétariat Permanent du Comité National de Lutte contre la Pratique de l'Excision \\
SR & Santé de la Reproduction \\
SRAJ & Santé de la Reproduction des Adolescents et Jeunes \\
SSR & Santé Sexuelle et Reproductive \\
UERD & Unité d'Enseignement et de Recherches Démographiques \\
UNICEF & Fonds des Nations Unies pour l'enfance \\
\hline
\end{tabular}




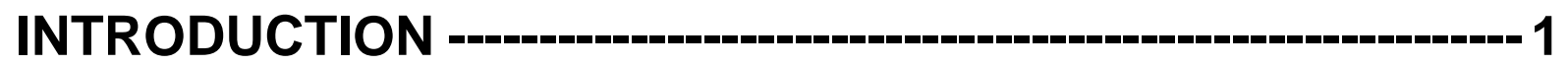

L'ENVIRONNEMENT INSTITUTIONNEL ET POLITIQUE ------ 2

LES CONVENTIONS INTERNATIONALES ET TEXTES NATIONAUX --:--:--:--:-----3

L'HISTORIQUE DE LA LUTTE CONTRE L'EXCISION AU BURKINA FASO ---:-----3

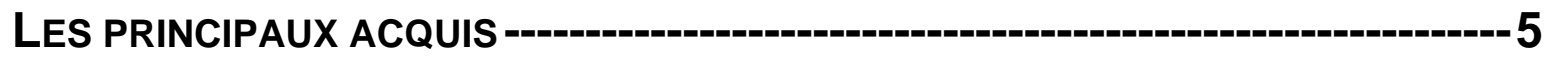

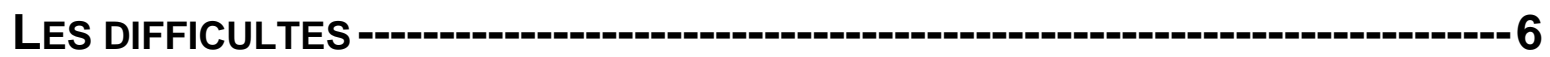

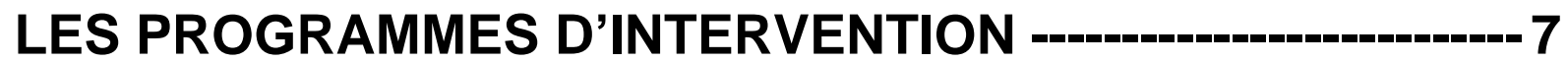

Le Comite national de Lutte contre la Pratique de l'Excision

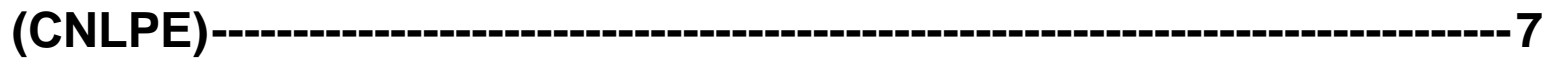

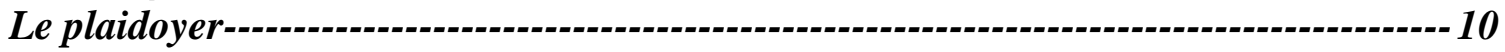

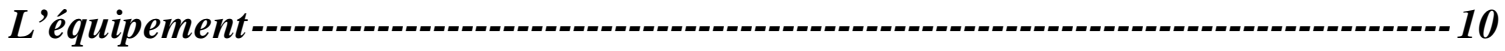

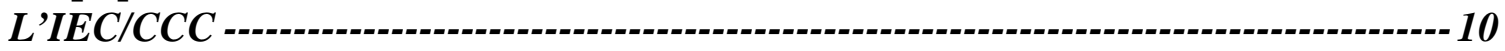

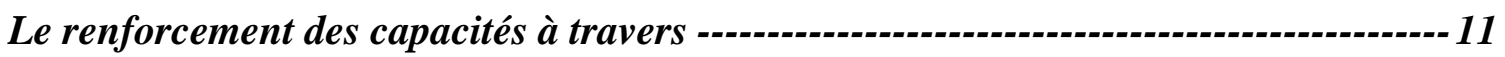

La réparation des cas de séquelles d'excision-------------------12

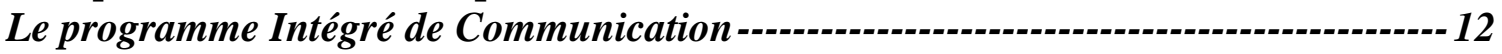

Les patrouilles de sensibilisation et de dissuasion-----------------------------14

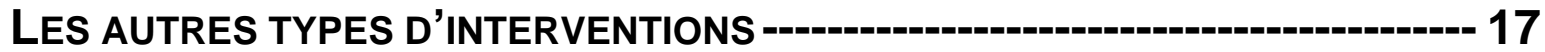

L'approche holistique par l'éducation aux droits humains---------------------17

L'approche des Imams----_e

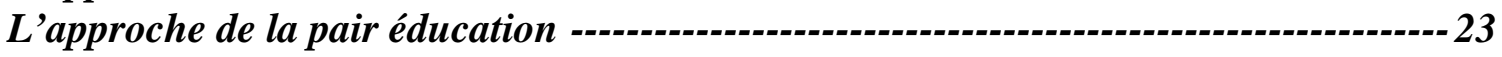

L'approche des leaders communautaires ---------------------------------------- 25

L'approche de l'intégration des modules dans le cursus scolaire ---------------------27

Le Projet Mille Jeunes Filles ------------------------------------------- 29

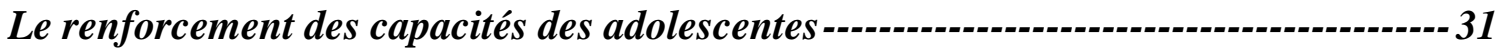

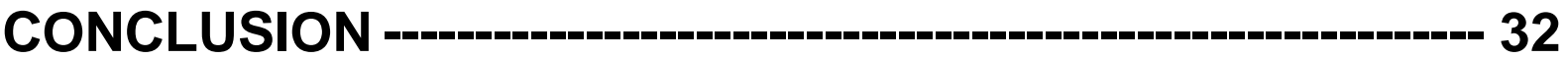

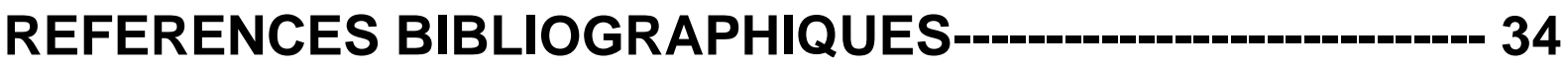




\section{Introduction}

L’OMS estime à 2 millions ${ }^{1}$ les fillettes menacées chaque année de subir une forme ou une autre d'excision (Mutilations Génitales Féminines). En Afrique, l'excision est pratiquée dans 28 pays y compris le Burkina Faso où la forme la plus répandue l'ablation du clitoris et des petites lèvres.

Au Burkina, l'excision touche presque toutes les ethnies et est pratiquée sur l'ensemble du territoire. L'analyse secondaire des données d'enquêtes réalisées depuis l'adoption de la loi, montre une tendance en dents de scie dans l'évolution de la pratique. En 1996, la prévalence de l'excision était estimée à 66\% chez les femmes de 15-49 ans (INSD 1996). Ces données de l'EDS (2003) établissent un taux national de 77\% de prévalence au sein des petites filles (0-4 ans). Pourtant, dans certaines provinces comme le Bazéga, la prévalence de l'excision au sein du même groupe d'âge de filles est estimée à moins de $1 \%$. Cette situation illustre donc une disparité de situations suivant les zones.

Plusieurs conventions et plans d'actions internationaux ont condamné l'excision en tant que violation des droits de la personne humaine, des droits de l'enfant et du droit à la santé et à l’intégrité physique. En adhérant à ces différentes conventions, le Burkina Faso a fait de la lutte contre la pratique de l'excision une de ses priorités.

La présente analyse fait un tour historique des événements majeurs de la lutte contre la pratique avant d'aborder les conventions internationales auxquelles le Burkina a adhéré ainsi que les textes nationaux adoptés en ce qui concerne les droits de l'homme, spécifiquement le droit des femmes et des enfants. Elle analyse également l'application de la loi à travers l'évolution des cas de condamnation depuis l'adoption de la loi. Enfin, elle s'intéressera à quelques programmes d'intervention mis en place en identifiant les acquis et les limites. Ce travail fait partie d'une étude sur l'évolution de la pratique de l'excision. Elle est complétée par une analyse secondaire des données ${ }^{2}$, et une enquête qualitative dans cinq provinces ${ }^{3}$.

\section{Méthodologie}

L'information a été recueillie par la combinaison de la revue documentaire et de l'interview individuelle. La revue documentaire a consisté à collecter les documents relatifs aux droits internationaux sur les femmes et les enfants que le Burkina a signé ou ratifié ainsi que les textes nationaux sur le même sujet. Dans un second temps, il s'est agit de collecter les documents et rapports d'étude et d'évaluation de quelques programmes d'intervention sur l'excision.

Des interviews individuelles ont été menées avec des responsables de programmes et des personnes ressources de structures nationales. Dans la plupart des cas, les interviews individuelles ont été enregistrées, avec l'accord du répondant. Pour chaque type de répondant, des instruments ont été élaborés. Des enquêteurs ont été recrutés pour collecter les données.

\footnotetext{
${ }^{1}$ OMS, 1995.

${ }^{2}$ Analyse de l'évolution de la pratique de l'excision au Burkina à partir des données existantes, janvier 2006.

${ }^{3}$ Analyse des perceptions et comportements de la population au Burkina, septembre 2006.
} 
Tableau 1 : Personnes interviewées au niveau des institutions

\begin{tabular}{|c|c|c|c|c|c|c|c|}
\hline \multicolumn{8}{|c|}{ Personnes interviewées } \\
\hline Institutions & MASSN & $\begin{array}{l}\text { Ministère } \\
\text { de la santé }\end{array}$ & $\begin{array}{l}\text { Force } \\
\text { sécurité }\end{array}$ & $\begin{array}{l}\text { Ministère } \\
\text { de la } \\
\text { justice }\end{array}$ & $\begin{array}{l}\text { Ministère de } \\
\text { l'éducation }\end{array}$ & $\begin{array}{l}\text { Autres } \\
\text { partenaires }\end{array}$ & ONGs \\
\hline & 4 & 3 & 9 & 3 & 4 & 3 & 4 \\
\hline
\end{tabular}

En effet, comme le récapitule le tableau ci-dessus, des interviews ont été réalisés auprès des institutions suivantes : Ministère de l'Action Sociale et de la Solidarité Nationale (MASSN), Ministère de la Santé, de la justice, La Direction de l'Education en matière de population, le programme de santé sexuelle et de droit humain, ainsi qu'auprès des ambassades du Danemark, du Pays bas, au niveau de la police et de la gendarmerie nationale (au niveau de Bobo, Ouahigouya et Ouaga) au niveau des responsables d'associations luttant contre l'excision, et au niveau également des ONGs s'occupant de ces questions.

Trois tribunaux ont été visités pour relever les condamnations d'excision. Il s'agit des tribunaux de grande instance de Ouagadougou, Bobo-Dioulasso et Ouahigouya.

Au niveau de l'analyse documentaire, le contenu des programmes a été ciblé, ainsi que les acteurs, l'intensité et à la durée de l'intervention, les groupes cibles, le mode d'évaluation du succès, les indicateurs de succès de l'intervention, l'impact du programme, les principaux résultats atteints, les défis et difficultés rencontrées et enfin les solutions et stratégies apportées.

En ce qui concerne les guides d'entretien avec les responsables de programme, la première étape de l'entretien retrace les méthodes utilisées telles que le plaidoyer politique et la mobilisation communautaire, l'évaluation des activités, l'impact de la loi dur la pratique de l'excision, leur collaboration avec les ministères, leur perspective pour une lutte plus efficace contre la pratique ainsi que leurs recommandations à l'endroit du CNLPE pour des actions plus efficace.

\section{Difficultés}

L'insuffisance de disponibilité des acteurs de terrain. Les activités menées sur le terrain ont eu lieu pendant les mois d'août et de septembre. Cette période étant celle des congés pour la plupart des responsables de programme, il a été difficile de les rencontrer afin d'échanger sur la question.

L’absence d'un système d'archivage des documents (rapports d'activités, de recherche).

L'absence de rapports d'évaluation dans certains cas n'a pas permis de disposer des informations à exploiter, notamment les acquis, les difficultés et les recommandations formulées pour une meilleure efficacité des actions.

\section{L'environnement institutionnel et politique}

L'environnement institutionnel et politique actuel de la lutte contre l'excision au Burkina résulte de la combinaison des actes administratifs et du cadre juridique national et international dans lequel s'inscrit le pays. Ce cadre administratif et juridique crée, consolide et favorise des actions directes de lutte contre la pratique de l'excision. 


\section{Les conventions internationales et textes nationaux}

Le Burkina Faso a ratifié des conventions internationales et adopté des textes au niveau national en faveur des droits de la femme et des enfants (tableau 2).

\begin{tabular}{|l|l|}
\hline \multicolumn{2}{|c|}{ Tableau 2: Conventions et textes adoptés par le Burkina Faso } \\
\hline Les conventions et textes nationaux & $\begin{array}{l}\text { Date } \\
\text { ratification } \\
\text { /adoption }\end{array}$ \\
\hline Conventions régionales et internationales & 1984 \\
\hline La charte de Banjul & 1987 \\
\hline La convention sur les droits des femmes & 1990 \\
\hline La convention sur les droits des enfants & 1992 \\
\hline La charte africaine & 1999 \\
\hline La convention sur les droits civils et politiques & 1999 \\
\hline La convention sur les droits culturels, économiques et sociaux & 1979 \\
\hline La charte africaine sur les droits de l'enfant & 2005 \\
\hline $\begin{array}{l}\text { Le protocole à la charte africaine des droits de l'homme et des peuples relatifs aux droits } \\
\text { des femmes (protocole de Maputo) }\end{array}$ & \\
\hline Textes nationaux & 1991 \\
\hline La constitution du Burkina Faso & Novembre 1996 \\
\hline La loi condamnant la pratique de l'excision &
\end{tabular}

\section{L'historique de la lutte contre l'excision au Burkina Faso}

L'environnement favorable à la lutte contre l'excision au Burkina est marqué par un certain nombre d'actions entreprises depuis la période coloniale.

Dans les années 1900, pendant la colonisation, les missionnaires catholiques ont tenté de mettre fin à la pratique en interdisant aux fidèles chrétiens de pratiquer l'excision, sous peine de se voir excommunier. Cela a entraîné le refus de certains fidèles tandis que d'autres ont adopté la nouvelle mesure. Cependant, ce nouveau groupe de filles a par la suite été confronté au délicat problème de trouver un mari. Face à cette situation, certaines familles ont entrepris de faire exciser leurs filles juste avant le mariage, d'autres ayant fait exciser la fille une fois dans le foyer conjugal. Préoccupés à garder leurs croyants, les missionnaires ont mis fin à leur injonction à ne pas exciser les filles.

En 1960, la première république de Haute Volta a essayé d'interdire la pratique mais s'est vu confronté à une résistance farouche des dignitaires du régime et de la chefferie traditionnelle et coutumière. Au regard de l'engagement de ces différents groupes sociaux et des forces politiques, cette action de lutte a été vite abandonnée.

En 1975, la célébration de la journée internationale des femmes a été un tournant majeur dans l'engagement du pays dans la lutte en faveur de l'abandon de l'excision. Elle a été particulièrement marquée par la toute première annonce dans la presse, notamment la radio nationale, d'informations tendant à discréditer la pratique de l'excision. Cette annonce n'a pas connu de suite face aux réticences de la population en général et la forte pression sociale que toute la famille de l'annonceuse a subi.

En Avril 1977, le mouvement international «Terre des hommes » lance depuis Genève une campagne de grande envergure visant à dénoncer l'excision en interpellant par lettre l'organisation des nations unies. C'est lors de cette campagne que Le 20 décembre 1983, Mr. 
Edmond Kaiser a rencontré Mr Thomas SANKARA, le président du Burkina, sur la question de l'excision. Le chef de l'Etat avait promis de faire une déclaration sur la question à travers les ondes ${ }^{4}$. La volonté affichée des premières autorités du pays pour l'émancipation et la protection de la femme a été un tournant décisif dans l'acceptation de la promotion de l'abandon de l'excision comme un problème qui mérite une attention toute particulière.

En 1985, un séminaire national a été organisé par l’Union des Femmes Burkinabé (UFB). Ce séminaire avait pour but de parler des questions liées à la femme, parmi lesquelles il y avait l'excision. Au cours de ce séminaire, les femmes ont demandé l'adoption de textes interdisant purement et simplement la pratique de l'excision.

De 1985 à 1988, l’ONG Sentinelles a accompagné la réflexion entreprise par l’Union des Femmes Burkinabé et a établi des contacts avec un groupe de militants autour de la question de l'excision. A l'issu de ces contacts, le Ministère de l'Essor familial a été touché en vue de l’organisation d'une réflexion nationale sur la question.

En 1988, un séminaire d'envergure nationale est organisé sur « les pratiques traditionnelles néfastes à la santé des femmes : le cas de l'excision ». Ce séminaire a eu lieu dans le plus grand stade de football de la capitale et a connu la participation de plus de 300 délégués venus de toutes les provinces et de toutes les couches ou catégories de la population. Il a été relayé par les différents médias : radio, télé, presse écrite. Ce séminaire marque le début de la formalisation de la lutte contre la pratique. Trois recommandations sont sorties de cette rencontre: (i) la mise en place d'un cadre institutionnel chargé de la lutte; (ii) la sensibilisation; (ii) l'implication des leaders communautaires et religieux. Le comité d'organisation de l'atelier a été érigé en comité provisoire du comité national et a commencé à travailler dans dix provinces identifiées comme étant les plus touchées par la pratique. En 1989, le même séminaire est organisé à Ouahigouya à une échelle plus réduite au niveau provinciale.

Le 18 mai 1990, le Comité National de Lutte contre la Pratique de l'Excision (CNLPE) est né sous un décret présidentiel (Kiti nAN VII-318/FP/SAN-AS/SEAS) avec des démembrements dans les provinces. Il est composé de 60 membres nommés par décret présidentiel dont 13 ministères, plusieurs ONG et associations (féminines, professionnelles, des droits de l'homme, etc.), des leaders religieux, coutumiers ainsi que des personnes ressources. La présidence d'honneur est assurée par l'épouse du chef de l'Etat. Le comité est décentralisé avec des démembrements au niveau provincial, départemental et villageois.

En 1992, un premier plan d'action est adopté pour la période 1993 à 1997. Il définit clairement les activités à mettre en place. Sur la base de ce document, une première table ronde des bailleurs de fonds est organisée sur la question en 1994. Elle a permis d'attirer l'attention de ceux-ci sur le problème de l'excision et suscité leur engagement pour le financement des activités.

En 1996 : Adoption de la loi réprimant la pratique de l'excision. En la faveur de la relecture du code pénal burkinabé, des actions de plaidoyer ont été initiées dans le but d'intégrer des articles dans le nouveau code pénal. Il s'agissait dans un premier temps de convaincre les membres du ministère de la justice, ensuite ceux du comité de relecture et enfin les députés à

\footnotetext{
${ }^{4}$ Le président Thomas Sankara disait en substance ceci dans l’extrait de la bande enregistrée : « Je puis vous assurer que je m’adresserai aux Voltaïques dans ce sens, et tant mieux si la voix d'un Voltaïque, la mienne, si cette voix-là peut aller au-delà de nos frontières. Parce que nous estimons que c'est un combat juste, c'est un combat noble que vous menez » (Sentinelles : les mutilations sexuelles féminines et leur abolition, une lutte africaine). On ne sait malheureusement pas si le président a réellement fait cette déclaration
} 
l'assemblée nationale. Certains députés avaient déjà bénéficié d'informations au travers de séminaires de sensibilisation organisés à leur intention. Ce qui a accéléré l'adoption de la loi à l’Assemblée Nationale.

En 1997, le comité national de lutte contre la pratique de l'excision se dote d'un secrétariat permanent qui est chargé de la mise en œuvre d'un certain nombre d'activités. Le gouvernement y affecte des agents de l'Etat (y compris l'engagement de la force publique). Depuis 2000, l'excision est considérée comme faisant partie du programme d'investissement public et le budget de fonctionnement du secrétariat permanent est pris en compte dans le budget de l'Etat afin d'assurer une disposition de personnel permanent. La même année, on assiste au début de la mise en œuvre de la loi avec les premières condamnations. Cependant, ces condamnations ont un caractère de sensibilisation et ne comportent pas de peines de prison. Il s’agit principalement de condamnation avec sursis.

Depuis 2000, la date du 18 mai est consacrée comme journée nationale de lutte contre l'excision dont la première a été célébrée en 2001. L’excision est désormais considérée comme un problème de santé publique par le ministère de la santé et intégrée dans la formation du personnel de santé.

\section{Les articles du code pénal condamnant l'excision}

Les trois articles suivants sont inscrits au chapitre II des crimes et délits contre la famille et les bonnes mœurs:

- Article 380

Est puni d'un emprisonnement de 6 mois à 3 ans et d'une amende de 150.000 à 900.000 francs ou de l'une de ces deux peines seulement, quiconque porte ou tente de porter atteinte à l’intégrité de l'organe génital de la femme par ablation totale, par excision, par infibulation, par insensibilisation ou par tout autre moyen.

Si la mort en est résultée, la peine est un emprisonnement de 5 à 10 ans.

- Article 381

Les peines sont portées au maximum si le coupable est du corps médical ou paramédical. La juridiction saisie peut en outre prononcer contre lui l'interdiction d'exercer sa profession pour une durée qui ne peut excéder 5 ans.

- Article 382

Est puni d'une amende de 50.000 à 100.000 francs, toute personne qui ayant connaissance des faits prévus à l'article 380 n'en avertit pas les autorités compétentes.

\section{Les principaux acquis}

\section{La collaboration entre ministères}

Treize ministères sont membres du comité national de lutte contre l'excision (CNLPE). Une collaboration étroite est assurée avec le ministère de la défense et celui de la santé. Un gendarme représentant le ministère au sein du CNLPE et un agent de santé sont détachés au sein du Secrétariat Permanent du comité (SP/CLPE) pour accompagner et coordonner les 
interventions de la gendarmerie et de la police en cas d'intention ou de cas d'excision signalé et/ou constaté. Avec le ministère de la santé, deux gynécologues assurent la formation des autres gynécologues et autres médecins aux techniques de réparation mais accueillent également les patientes souffrant de séquelles. Les ministères de l'éducation et de l'information jouent pleinement leur rôle en introduisant cette problématique dans leurs activités et curricula.

\section{L'engagement des groupes socio-professionnels}

Les agents de santé ont été formés dans la reconnaissance des séquelles de l'excision et en techniques de réparation. Les magistrats ont été sensibilisés sur la question de l'excision et encouragés à appliquer la loi de façon rigoureuse, en évitant surtout les nombreux sursis constatés. Les forces de sécurité et de police ont été sensibilisés à la question et formés pour intervenir sur les cas avérés d'excision. Plusieurs ONGs et associations communautaires sont membres du CNLPE de même que des représentants de la société civile (représentant des jeunes, des coutumiers et des traditionnels, des associations et groupes islamiques, des religieux chrétiens, des médias, ...).

\section{L'engagement des groupes sociaux}

En 1995, un séminaire est organisé avec les leaders communautaires et religieux. A l'issue de ce séminaire, les groupes ont été sensibilisés de façon séparée pour une meilleure efficacité. Ces séances de sensibilisation ont eu pour effet de susciter un engagement des leaders par la mise en place de comités des chefs religieux musulmans (1996), catholiques (1997), des chefs coutumiers (1996).

\section{Les appuis des ONGs et des partenaires au développement}

Le lobbying que mène la structure a permis une adhésion effective des partenaires au développement. Cette adhésion se traduit en tout temps par des actions et des appuis multiformes qui permettent la mise en œuvre des activités. Les partenaires techniques et financiers qui appuient le CNLPE sont : UNICEF, OMS, UNFPA, Banque Mondiale (PPLS), Ambassades Royales des Pays - Bas et de Danemark, Plan International, GTZ, Centre Canadien d'Etudes et de Coopération Internationale, Aide à l'enfance Canada, RAINBO, CIAF, Population Council, USAID, WIN, GAMS, Public Welfare Fundation, Coopération Suisse, Oxfam Québec, Sentinelles, Medicus Mundi, ...

Beaucoup d'autres activités sont menées sur le terrain par les associations, les ONGs, les partenaires financiers. Dans certains cas les activités sont centrées sur la pratique de l'excision, dans d'autres, elles concernent des aspects plus larges de santé de la reproduction, englobant ainsi les questions liées à l'excision.

\section{Les difficultés}

\section{Les réactions des populations}

La difficulté d'aborder de front la question de l'excision a conduit à trouver une porte d'entrée. C’est pourquoi la question a été abordée sous le volet santé. Le principal sujet abordé en premier était la planification familiale et des autres préoccupations des populations, notamment celles des femmes. La question de l'excision n'était abordée que lorsque l'environnement et le climat qui entourait l'auditoire en étaient favorables. Il n'était jamais prévisible de savoir si la question de la pratique de l'excision serait abordée à cause des agressions verbales et mêmes physiques : les femmes qui faisaient la sensibilisation étaient traitées de femmes faciles, dévergondées, prostituées. 
Les premiers moments des interventions furent difficiles parce qu'il n'y avait pas de cadre juridique pour s'attaquer au phénomène. Les plus farouches opposants à la lutte furent les femmes qui s'insurgeaient contre le principe de la lutte mais également contre les méthodes utilisées : présentation dans les séances de sensibilisation et à la télévision de mannequin de femme nue.

Au niveau politique, il n’y avait pas vraiment d'opposition ou de désapprobation ouverte. Cependant, on signale quelques interventions de personnes influentes (hommes politiques, etc.) pour faire libérer des personnes condamnées ou pour les faire condamner avec sursis. Les premières condamnations étaient essentiellement des condamnations avec sursis. A l'issue de ce constat, un séminaire de sensibilisation a été organisé en direction des magistrats pour une meilleure connaissance des conséquences de la pratique.

\section{Les programmes d'intervention}

Depuis une dizaine d'années, plusieurs approches ont été appliquées au Burkina dans le but de contribuer à l'élimination de la pratique de l'excision. Ces approches différentes peuvent être analysées en terme de contenu, de zone d'intervention, de public cible, d'acquis et de limites.

\section{Le Comité National de Lutte contre la Pratique de I'Excision (CNLPE)}

Le CNLPE est chargé de :

- La réalisation d'études et recherches en vue d'acquérir une connaissance adéquate du phénomène ;

- La collecte et la diffusion de toutes les données disponibles relatives aux pratiques affectant la santé des femmes et des enfants ;

- L'élaboration, la mise en œuvre et le suivi-évaluation de toutes les stratégies de lutte visant l'abolition progressive de la pratique de l'excision et de toutes les autres formes de pratiques affectant la santé et l'épanouissement des femmes et des enfants ;

- La promotion d'activités de sensibilisation, d'éducation et d'information sur toutes ces pratiques ;

- La mobilisation des ressources matérielles et financières à la lutte contre toutes les pratiques affectant la santé et l'épanouissement des femmes et des enfants.

Au début de l'année 1997, le CNLPE s'est doté d'un Secrétariat Permanent (SP/CNLPE) pour rendre la structure plus fonctionnelle et plus opérationnelle. Ce secrétariat permanent est chargé de (i) la préparation des réunions et des documents du CNLPE ; (ii) la mise en œuvre et le suivi des décisions du CNLPE ; (iii) l'élaboration et la mise en œuvre du plan d'action national ; (iv) la gestion administrative et financière du CNLPE ; (v) la coordination des activités des structures décentralisées (CPLPE et CDLPE). Le gouvernement y affecte des agents de l'Etat (y compris l'engagement de la force publique) afin d'assurer la disposition d'un personnel permanent. 


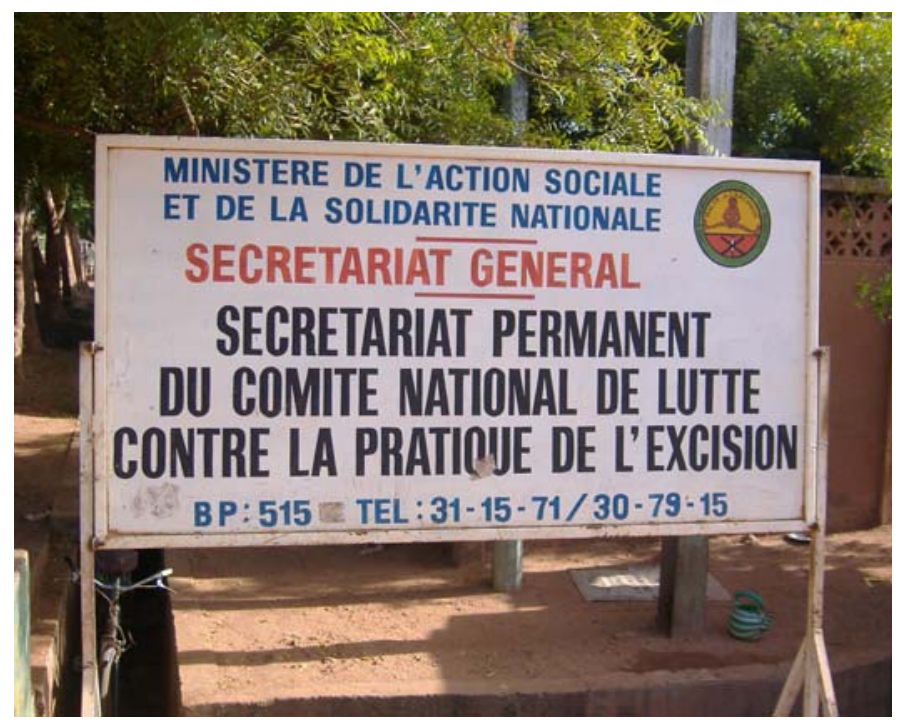

Siège du Secrétariat Permanent

L’organigramme du CNLPE

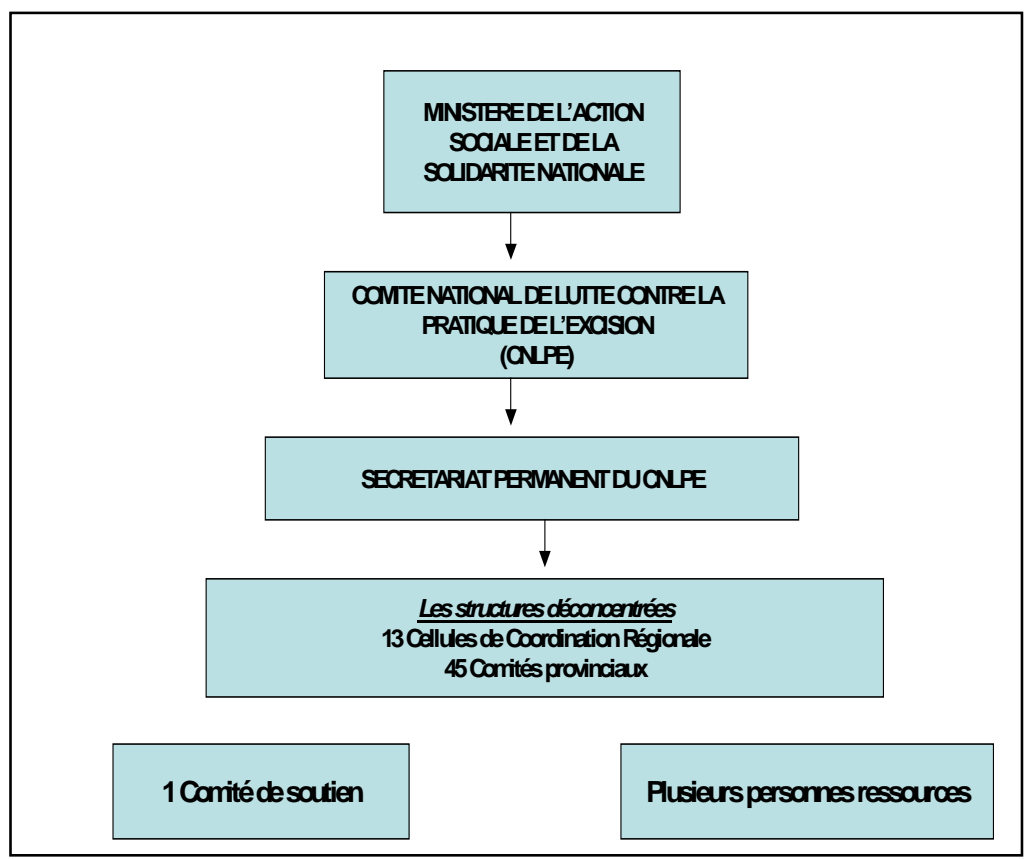

Le CNLPE est sous la présidence d'honneur de la première dame du Burkina et sous la responsabilité de la direction du Ministère de l'Action Sociale et de la Solidarité Nationale. Puis viennent le Secrétariat Permanent, ensuite les structures décentralisées composées de 13 cellules de Coordination Régionale et de 45 Comités provinciaux. Enfin, il existe un comité de soutien et les personnes ressources. 


\section{Le téléphone vert}

Le téléphone a été un moyen au début des interventions pour les gens pour insulter et dénoncer les efforts de lutte contre la pratique. Il est donc apparu opportun d'utiliser ce même canal pour dénoncer non pas les actions de lutte mais plutôt les cas éventuels ou avérés d'excision. C'est ainsi que le téléphone "S.O.S Excision" a été mis en place et est pris entièrement en charge par le budget de l'Etat. Aujourd'hui, le CNLPE est doté d'un téléphone vert qui permet de signaler de façon anonyme toute information susceptible de prévenir ou d'appréhender des auteurs et complices d'excision sans frais pour l'appelant. Cependant, il n'existe pas de moyens d'évaluation de la rentabilité du téléphone vert. Il devrait y avoir un moyen de savoir le nombre d'appels reçus, le nombre de cas réellement traités suite à un coup de fil.

\section{L’application de la loi contre l'excision}

D’août 2005 à octobre 200621 cas ont été enregistrés concernant plus d'une trentaine de fillettes (dans les provinces du Yatenga, de l'Oubritenga, du Poni, du Kadiogo, de la Leraba, des Balé, du Sanmatenga, du Boulgou, du Kourweogo, de la Comoé, et du Bam). Plus de 36 personnes ont été interpellées par la justice. Dans la majorité des cas, il s’agit de l'exciseuse, de ses complices, et souvent les parents de la victime.

Tableau 3 : Quelques données sur les condamnations dans trois Tribunaux

\begin{tabular}{|c|c|c|c|c|c|c|c|}
\hline & & & \multicolumn{5}{|c|}{ Condamnations } \\
\hline $\begin{array}{l}\text { Tribunal de } \\
\text { Grande } \\
\text { Instance (TGI) }\end{array}$ & Période & $\begin{array}{l}\text { Nombre } \\
\text { de pers }\end{array}$ & $<6$ mois & $\begin{array}{l}6 \text { mois } \\
-1 \text { an }\end{array}$ & 1 an + & $\begin{array}{l}\text { Données } \\
\text { manquantes }\end{array}$ & CSS \\
\hline Ouahigouya & 2004-2005 & 21 & 18 & $\mathbf{0}$ & $\mathbf{0}$ & $\mathbf{0}$ & 3 \\
\hline Ouaga & 2000-2003 & 17 & 6 & 1 & 4 & 6 & $\mathbf{0}$ \\
\hline Bobo & 1997-2005 & 56 & 3 & 10 & 5 & 38 & $\mathbf{0}$ \\
\hline Total & 1997-2005 & 94 & 27 & 11 & 9 & 44 & 3 \\
\hline
\end{tabular}

Bien que de moins en moins de cas signalés échappent à la justice, plusieurs condamnations ont été inférieures aux 3 mois stipulés dans la loi ou sont des condamnations avec sursis. On note également de nombreuses interventions (personnes influentes, proches parents, ...) pour soit éviter la condamnation, soit faire condamner avec sursis ou soit faire libérer les exciseuses, les parents et/ou les complices. Dans certains cas, le dossier est classé sans suite tandis que dans d'autres situations la libération intervient avant l'épuration de la peine. Toutes ces actions ne sont pas de nature à rassurer ceux qui ont décidé de respecter la loi. Sur les données disponibles dans les trois juridictions visitées ${ }^{5}$, plusieurs personnes ont été condamnées à 1 mois de prison. Sur 94 personnes condamnées, 27 ont eu une peine de moins de 6 mois tandis que 3 ont eu un dossier classé sans suite.

\footnotetext{
${ }^{5}$ Les tribunaux de grande instance de Ouagadougou, Bobo-Dioulasso et Ouahigouya
} 


\section{Le plaidoyer}

Les activités du plaidoyer sont dirigées vers les leaders religieux et coutumiers, les parlementaires, les responsables de groupements et associations à travers tout le pays. Il s'agit de rallier à la cause de la lutte tous les leaders susceptibles d'influencer les attitudes et les comportements des populations, notamment les populations analphabètes. Au delà des objectifs du plaidoyer, la sensibilisation est effectuée continuellement pour une implication effective des leaders politiques, administratifs, religieux, coutumiers et traditionalistes avec création de comités de soutien des chefs coutumiers, des chefs religieux (catholiques et musulmans).

Les activités du plaidoyer ont permis l'institutionnalisation de la journée du 18 mai de chaque année comme journée nationale de lutte contre la pratique de l'excision par décret $n^{\circ} 2001$ 258/PRES/PM/MASSN du 06 juin 2001, l'engagement de la société civile à travers l'implication des leaders d'opinion (coutumiers, religieux, traditionalistes), les Associations et les Organisations non gouvernementales qui oeuvrent à l'élimination de l'excision et la prise en compte du phénomène de sa pratique comme un problème de santé publique. Le plan d'action de lutte contre la Pratique de l'Excision est inscrit dans le programme d'investissement public du Ministère de l'Action Sociale et de la Solidarité Nationale.

\section{L'équipement}

L'adhésion effective des partenaires au développement (UNICEF, OMS, Banque Mondiale (PPLS), Ambassades Royales des Pays - Bas et de Danemark, Plan International, GTZ, Centre Canadien d'Etudes et de Coopération Internationale, Medicus Mundi) permettent par leurs appuis multiformes, la mise en œuvre de nombreuses activités (équipement des structures de lutte en moto, matériel audio - visuel, groupes électrogènes, supports d’animation, en matériel bureautique etc. ).

\section{L'IEC/CCC}

L'information éducation communication/communication pour le changement de comportement (IEC/CCC) est une stratégie développée depuis bien longtemps dans le but de distiller des informations utiles à un groupe ou public cible. Dans sa stratégie opérationnelle de lutte contre la pratique de l'excision, le CNLPE a adopté la sensibilisation comme méthode privilégiée en raison du fait que la majorité de la population méconnaît les méfaits de l'excision. Plusieurs activités rentrent dans le cadre de cette stratégie. Il s’agit notamment de :

- La production et la diffusion de supports : la confection et la distribution de supports (affiches, dépliants, guides d'animation) en plusieurs langues, la confection de Tshirts, des projections de films, de gadgets aux effigies avec ou sans message sur l'abandon de la pratique ;

- La réalisation et la diffusion de spots publicitaires (radio, télé et journaux);

- La réalisation et la mise en place d'activités de sensibilisation telles que les films, les théâtres forum, les causeries classiques, les causeries débat, etc ;

- La mise en place d’une ligne téléphonique dénommée "SOS/Excision"pour établir un contact permanent avec les populations ;

- La création d'un Site Web qui permet au CNLPE de diffuser à grande échelle les données collectées et de se faire connaître à travers le monde ;

- La création d'un centre de documentation afin de mettre à la disposition des usagers un centre de recherche sur les violences, les droits des femmes et des enfants ainsi qu'une banque de données bibliographiques, plus spécifiquement les Pratiques Traditionnelles Néfastes et les Mutilations Génitales Féminines. 


\section{Le renforcement des capacités à travers}

$\checkmark$ La formation des exciseuses et ex-exciseuses pour que celles-ci deviennent des relais de communication et de sensibilisation auprès de leurs consœurs exciseuses et auprès de leurs communautés respectives.

$\checkmark$ La formation d'agents de santé et d'accoucheuses villageoises pour la sensibilisation,

$\checkmark$ La formation de magistrats pour une plus grande application de la loi,

$\checkmark \mathrm{La}$ formation des agents sociaux en IEC/Excision et certains en suivi/évaluation, genre et lutte contre les violences faites aux femmes,

$\checkmark$ La formation des policiers et gendarmes pour les patrouilles de sensibilisation et les sorties préventives pour les cas de dénonciation d'intention d'exciser ;

$\checkmark$ La formation des élèves en IEC/excision pour des actions dans les établissements scolaires.
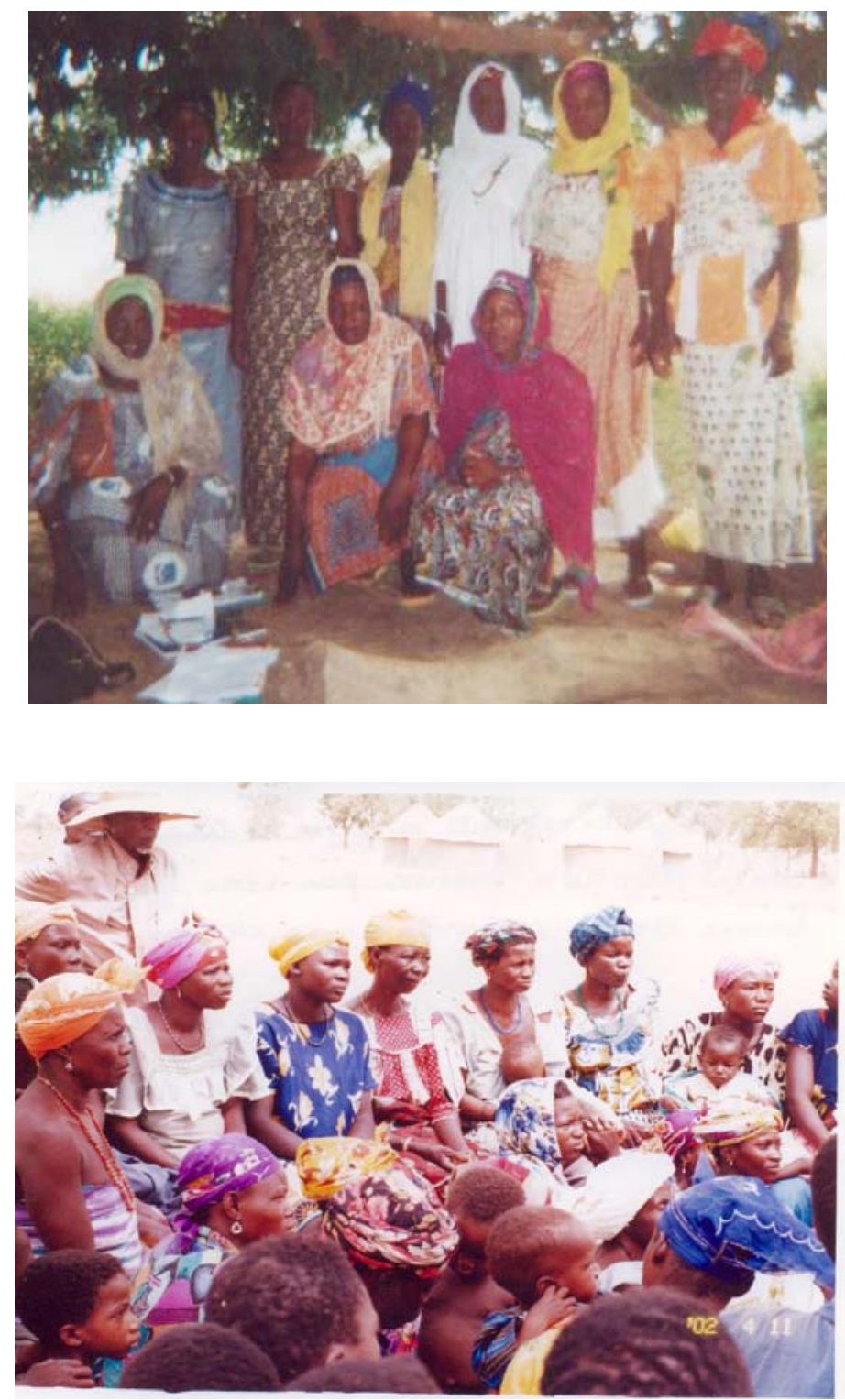

Réunion de sensibilisation des exciseuses et ex-exciseuses 


\section{Les acquis}

- Une meilleure visibilité des activités de lutte contre l'excision une démystification de l'excision (tabou levé, rôle des médias, présentation de photos choquantes, ...)

- Une application effective de la loi (responsables chargés de l'application de la loi mieux outillés).

\section{Les limites}

- Les campagnes de masse qui ne touchent pas forcément le public visé ;

- Le caractère unidirectionnel, ne tenant pas toujours compte de la remontée de l'information ;

- Les messages généraux, inadaptés aux différents contextes du pays et du stade d'évolution des zones ;

- Le faible accès à l'information pour les zones reculées, notamment celles qui n’ont pas accès à la couverture médiatique ;

- Le manque de traduction des informations dans plusieurs autres langues, notamment celles relatives aux zones les plus touchées.

\section{La réparation des cas de séquelles d'excision}

Pour pallier aux problèmes sanitaires des femmes et filles qui souffrent des séquelles de l'excision, le comité avec ses partenaires techniques et financiers ont initié et mis en œuvre la stratégie de réparation des cas de séquelles. Elle consiste à former des médecins gynécologues, des sages femmes des régions pour la réparation des séquelles et de doter les antennes chirurgicales de kit de prise en charge médicale.

\section{Les acquis}

- Une meilleure visibilité des activités de lutte contre l'excision,

- La création d'un environnement favorable à la déclaration des cas de séquelles,

- Une possibilité pour les victimes d'avoir une prise en charge médicale.

\section{Les limites}

- Le coût relativement élevé des prestations, notamment pour les plus démunis ;

- L’insuffisance de structures de prise en charge médicale des cas de complication ;

- L’insuffisance des médecins formés pour la prise en charge des séquelles ;

- Le manque de prise en charge psychologique, notamment pour celles qui souffrent dans leur foyer.

\section{Le programme Intégré de Communication}

Le Plan Intégré de Communication (PIC) est une approche axée sur la communication pour le changement de comportement, la mobilisation sociale autour de la question de l'excision et le plaidoyer. Ce programme met l'accent sur l'approche multimédia avec la communication radiophonique à travers la radio rurale et les radios communautaires, les troupes théâtrales pour les théâtres forum, les directions provinciales et les noyaux relais chargé de sensibiliser les populations à travers des Causeries débat et le porte à porte ainsi que les projections vidéo. 
L'objectif du PIC est de parvenir à des résultats qui se traduisent par le recul, voire l'abandon de la pratique de l'excision et amener les populations à se faire établir des actes d'état civil en l'occurrence les actes de naissance, les jugements supplétifs d'acte de naissance et les actes de mariage et des cartes d'identité. Le PIC dont l'expérimentation a commencé en 2001 dans la région du sud-ouest, s'est progressivement étendue à l'ensemble des zones couvertes par les différents partenaires.

Ce programme est soutenu par l'UNICEF et l'Ambassade Royale des Pays Bas. Les deux partenaires techniques et financiers soutiennent le CNLPE dans la mise en œuvre de ces activités dans chacune de leurs provinces d'intervention et au-delà. Dans le cas du Burkina, le PIC combine à la fois à savoir la lutte contre la pratique de l'excision et l'importance des actes d'état civil et de la carte d'identité burkinabé (CIB). Les provinces qui sont par contre couvertes par L’UNICEF sont actuellement au nombre de vingt deux.

L'Ambassade Royale des Pays-Bas intervient dans 16 provinces $^{6}$ au total dont 12 sont concernées par le PIC; les autres n'étant pas encore couvertes par une radio. Celles-ci bénéficient donc d'un appui aux associations locales pour des activités diverses liées à la sensibilisation pour l'abandon de la pratique.

La mise en œuvre du PIC comporte plusieurs volets :

$\checkmark$ Une approche multimédia avec la communication médiatique à travers des émissions en langue nationales, des animations dans les villages avec l'aide des stations radio,

$\checkmark$ Une communication interpersonnelle par des représentations théâtrales et des projections vidéo suivi de débats,

$\checkmark$ La mise en place de noyaux relais composés de six (06) à huit (08) personnes dans les villages et dans les secteurs permet de disposer d'un personnel permanent de sensibilisation dans les localités.

$\checkmark$ Des causeries et des portes à portes sont menées par les membres des mouvements associatifs villageois ou des quartiers.

\section{Les acquis}

- Forte implication des autorités administratives locales, adhésion des leaders d'opinion, responsabilisation des acteurs locaux à travers les relais communautaires ;

- Large couverture médiatique des messages sur l'excision ;

- Meilleure écoute des messages diffusés en langues locales ;

- Sensibilisation au quotidien avec les relais communautaires ;

- Forte implication des radios communautaires à la lutte contre la pratique ;

- Meilleure écoute.

\footnotetext{
${ }^{6}$ Kadiogo, Samnatenga, Namentenga, Oubritenga, Yatenga, Tapoa, Poni, Noumbiel, Bougouriba, Ioba, Comoe, Leraba

Les provinces couvertes par l’UNICEF sont: Kadiogo, Samnatenga, Namentenga, Oubritenga, Yatenga, Tapoa, Poni, Noumbiel, Bougouriba, Ioba, Comoe, Leraba, Kénédougou, Kouritenga, Ganzourgou, Seno, Zoundweogo, Nahouri, Bulkiemde, sanguie

Les provinces bénéficiant du PIC Pays Bas sont : Houet, Mouhoun, Kossi, Tuy, Zandoma, Boulgou, Kourweogo, Sanguie, Sissili, Bazega,

Loroum et du Passore. Les quatre autres provinces bénéficiant d’appui financier sont : Banwa, le Ziro, le Nayala et l'Oudalan.
} 


\section{Les limites}

- La période de diffusion des émissions n’est pas toujours adaptée au contexte local ;

- L’inadaptation de la langue de diffusion dans certaines zones de couverture ;

- Stratégie de masse, non centré sur un public cible spécifique ;

- Manque de suivi des activités des relais communautaires ;

- Manque de formation du personnel des radios ;

- Nécessite la mobilisation de moyens financiers énormes ;

- Faible mobilisation des relais communautaires choisis ;

- Faible contrôle du choix des noyaux communautaires ;

- Manque d'évaluation des activités ;

- Faible participation des femmes dans certaines régions (sahel);

- Le fait de confier la suite des activités à la population sans un diagnostic préalable des capacités cde ces zones. En effet, la population est sensée être mature pour prendre en charge la suite des activités tandis que les noyaux relais sont sensés être des leaders communautaires ayant une réelle influence pour apporter le changement souhaité. Dans la réalité, il n’a pas toujours été le cas.

\section{Les patrouilles de sensibilisation et de dissuasion}

Après l'adoption de la loi réprimant l'excision au Burkina Faso en 1996, le CNLPE en vue d'une application judicieuse de celle-ci a engagé des actions de sensibilisation, de formation et d'échanges avec les agents des forces de l'ordre (Gendarmes, Policiers), les Magistrats et les Avocats. A l'issue des différentes rencontres, les Brigades de Gendarmerie ont opté pour la réalisation de patrouilles de sensibilisation et de dissuasion comme stratégie à même de contribuer à l'élimination progressive de la pratique de l'excision au Burkina Faso.

A l'origine "la Patrouille" est une mission militaire donnée à une équipe légère motorisée ou pas, en vue de se porter isolément et en sûreté dans une zone donnée pour y accomplir une mission déterminée, regagner son point de départ et rendre compte. Ce concept a donc été adapté par les Brigades de Gendarmerie au contexte de la lutte contre la pratique de l'excision, de sorte que la patrouille de sensibilisation et de dissuasion se défini comme étant un ensemble d'activités spécifiques menées par les Gendarmes dans le cadre de la lutte contre la pratique de l'excision.

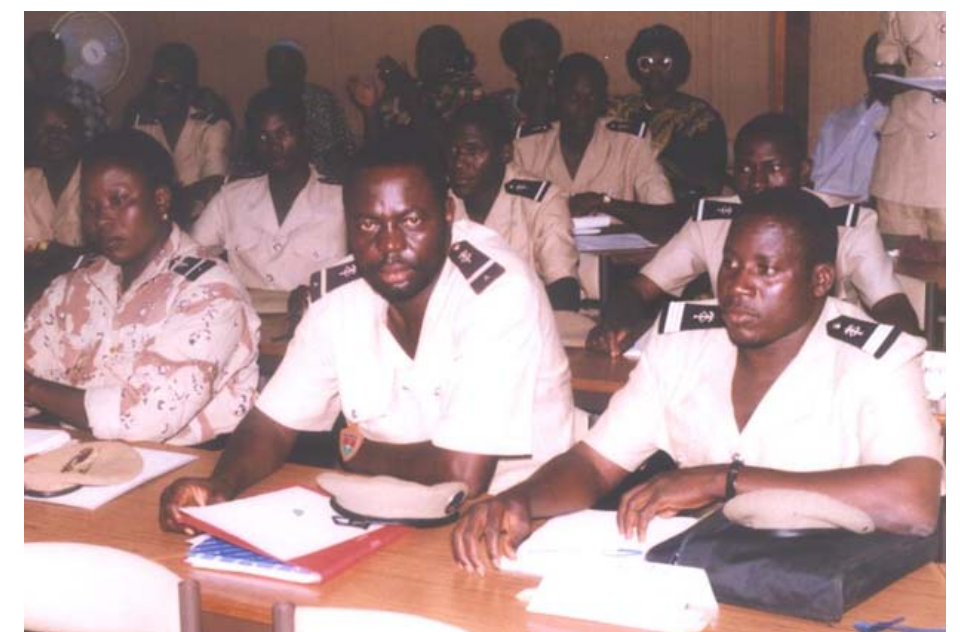

Formation de la brigade de gendarmerie en vu des patrouilles de sensibilisation et de dissuasion 


\section{La mise en ouvre}

Les patrouilles de sensibilisation et de dissuasion sont réalisées par vingt deux (22) Brigades de gendarmerie à travers seize (16) provinces ${ }^{7}$ les plus touchées par la pratique de l'Excision, selon les données de l’enquête nationale sur la pratique de l'excision réalisée en $1996^{8}$.

Pour permettre une meilleure coordination des Patrouilles, et de toutes les activités liées à l'application de la loi réprimant l'excision, une cellule d'intervention a été mise en place au sein du SP/CNLPE. Cette cellule d'intervention reçoit les appels pour les cas ou intentions d'excision, et organise selon la situation la descente des forces de l'ordre ou d'une équipe pluridisciplinaire sur le terrain.

Avant d'effectuer les patrouilles de sensibilisation et de dissuasion il faut au préalable faire une demande d'autorisation adressée au Chef d'État Major de la Gendarmerie Nationale avec précision de la période de réalisation souhaitée. Cette autorisation est accordée en fonction de la faisabilité de l'activité par les Brigades et de la période souhaitée au regard des missions du moment des brigades. La période favorable pour la réalisation des patrouilles de sensibilisation est la saison sèche c'est-à-dire du mois d'octobre au mois de mai et la réalisation des patrouilles couvre généralement une période de quatre (04) mois.

Chaque Brigade effectue une sortie par semaine, soit quatre (04) sorties par mois et ces patrouilles sont exécutées par un élément motorisé composé de quatre (04) gendarmes en tenue. Cet élément est chargé lors de sa sortie, de sensibiliser les populations dans les zones de grandes affluences (marchés, églises, temples, mosquées, casernes militaires, et autres lieux publics) sur différents thèmes relatifs à la pratique de l'excision, en mettant l'accent sur le contenu de la loi la réprimant.

Le commandant de Brigade est le responsable de l'activité. A ce titre il est chargé d'établir 48h à l'avance le bulletin de service qui donne l'ordre de mission et le rapport de mission après la sortie. Cet ordre de mission comporte les mentions suivantes : la date de sortie, la composition de l'équipe, la mission, les heures de départ et de retour et le secteur à visiter. En plus de ces mentions, le rapport après mission doit comporter les actions entreprises au cours de la patrouille, l'impact de la patrouille, les recommandations de la population, les suggestions des agents patrouilleurs et la conclusion. Une équipe du SP-CNLPE assure le suivi supervision des patrouilles.

Les activités réalisées par les brigades de gendarmerie sont :

- Les Causeries-débat (centrés sur les fondements, les conséquences de l'excision et le contenu de la loi réprimant l'excision) ;

- La vulgarisation de la loi à travers la distribution de brochure en français et en quatre langues nationales ;

- Le recensement et l'identification des exciseurs et exciseuses dans la zone ;

- La visite à domicile des exciseurs et exciseuses identifiés ;

- La mise en place d'un réseau d'informateurs et ;

- L'ouverture systématique d'une procédure judiciaire pour tout cas d'excision constaté.

\footnotetext{
${ }^{7}$ Ces 16 provinces constituent les provinces de la zone de couverture de l’UNICEF, qui seule finance les activités des patrouilles.

${ }^{8}$ INSD, 1996 : cette enquête nationale qui est la première du genre au Burkina avait identifié des provinces à prévalence élevée de pratique de l'excision. Elle a été suivie par la suite par un ensemble d'études parfois limitées à des zones spécifiques de travail
} 


\section{Les acquis}

La sortie des gendarmes pour faire de la sensibilisation et non pour réprimer a entraîner une meilleure communication et établie une certaine confiance avec les populations. Cette confiance entraîne une participation active et directe aux débats de toutes les couches sociales présentes pendant les sensibilisations. Ce qui permet aux gendarmes de faire passer le maximum d'informations sur l'excision, de donner des exemples concrets de procédures judiciaires et de convaincre les plus septiques que l'application de la loi est une réalité. Le recensement et l'identification des exciseurs et exciseuses permet d'assurer une surveillance continue sur ceux-ci et les visites à domicile des exciseurs et exciseuses identifiés permettent de les dissuader de pratiquer l'excision. La forte présence des gendarmes sur le terrain et la mise sur pieds discrète dans les villages d'un réseau d'informateurs pour dénoncer les cas ou intentions d'excision permettent de dissuader les populations de pratiquer l'excision.

Le nombre de cas d'excision a considérablement baissé dans les zones couvertes par les patrouilles de sensibilisation et de dissuasion; Plusieurs missions de pays voisins (consultants nationaux et étrangers, parlementaires maliens, membres de comités de lutte contre la pratique l'excision du Mali, du Togo et du Niger) viennent s’inspirer de l'expérience du Burkina de l'implication des forces de l'ordre dans la lutte contre la pratique de l'excision.

Le dispositif mis en place dans les villages lors des patrouilles permet d'espérer à moyen terme à un changement qualitatif de comportement vis-à-vis de la pratique de l'excision. La stratégie des patrouilles de sensibilisation et de dissuasion vient en complément aux autres stratégies mises en œuvre par le CNLPE.

\section{Les limites}

- Le manque de couverture de certaines provinces qui ne sont pas identifiées comme étant celles à forte prévalence mais ayant un niveau aussi élevé. Par conséquent, les populations de ces provinces ne bénéficient pas de cette stratégie et peuvent sentir moins la force de la loi ;

- La procédure administrative qui ne permet pas que les patrouilles aient lieu en temps voulu parce qu'ayant des missions spécifiques dévolues et régies par un autre ministère ;

- C'est surtout pendant la saison pluvieuse et celles des vacances que la pratique de l'excision est la plus forte, période qui ne correspond pas à celle des patrouilles de sensibilisation et de dissuasion ;

- La sortie des gendarmes focalise la lutte sur la loi et son application. Elle n'entraîne pas forcement une meilleure sensibilisation et peut exacerber les positions parce qu'apparentée à la force et à l'imposition, sans pour autant convaincre les populations du bien fondé de changer de comportement ;

- Le manque de formation ou de recyclage en IEC/Excision des agents patrouilleurs. Face aux interrogations des populations, les capacités de communication et de convictions réduites peuvent avoir un impact négatif sur les perceptions des populations ;

- Le manque de stratégie commune avec les pays voisins, qui aurait permis de poursuivre les contrevenants à la loi au-delà des frontières, en cas de déplacement transfrontalier des familles souhaitant exciser leurs filles ;

- Le manque d'évaluation des activités de ces patrouilles. Il apparaît alors difficile de mesurer l'impact réel des activités menées sur le terrain, aussi bien en termes d'amélioration des connaissances que de formation d'attitude positive et de changement de comportement ; 
- La persistance de la peur du gendarme au sein de la population qui entraîne une faible participation des femmes lors des sorties de sensibilisation. Dans ces conditions, la population féminine reste faiblement touchée par les messages délivrés ;

- Le manque de suivi régulier des sorties de terrain des brigades et des appels téléphoniques. Cela a pour conséquence de limiter l'évaluation de l'impact de ces deux différentes stratégies ;

- Le manque d'informations par rapport à l'efficacité des interventions suite aux appels reçus sur le numéro vert (nombre de filles sauvées...).

\section{Les autres types d'interventions}

Cinq provinces ont été identifiées comme étant des provinces où le niveau de prévalence de la pratique est très élevé. Avec l'appui de la GTZ un certain nombre de structures ou d’organisations locales qui y travaillent déjà ont été ciblées.

Avant la mise en œuvre des activités, une étude de base a permis de situer réellement la question de l'ampleur du phénomène et des facteurs explicatifs. Dans le même temps, une étude diagnostique a permis de recenser les structures et organisations au niveau local susceptible de développer et mettre en œuvre des stratégies de lutte efficaces contre la pratique de l'excision. L'étude de base ainsi que l'évaluation à mi-parcours et l'évaluation finale ont permis de disposer des informations aussi bien quantitatives que qualitatives, malgré le fait que cela n’a pas été systématique pour toutes les approches utilisées.

\section{L'approche holistique par l'éducation aux droits humains}

Face aux insuffisances des approches basées sur la répression et les aspects sanitaires, ce programme propose une approche globale qui s'intéresse à plusieurs aspects du développement aussi bien humain que socio-économique. Il met l'accent sur le développement social intégral.

\section{Mise en oeuvre}

La mise en place du programme d'intervention a nécessité le développement d'une démarche de mobilisation sociale. Cette démarche s'articule autour de cinq phases: la phase préparatoire, la phase d'orientation, la phase d'organisation, la phase de mise en œuvre et la phase d'évaluation et de dissémination.

La phase préparatoire a consisté dans un premier temps à la traduction en Mooré ${ }^{\text {, du }}$ curriculum de $\operatorname{Tostan}^{10}$ et à la formation de l'équipe d'encadrement. Cette équipe d'encadrement, composée de 2 chargés de programme de Mwangaza et de 3 superviseurs a effectué un séjour d'un mois à Thiès (au Sénégal) pour une formation par l'équipe de l'ONG Tostan. La participation de cette équipe à la déclaration publique de promotion de la femme de Aérélao et de Karcia a permis à ces responsables de s'imprégner du processus organisationnel et d'animation d'une telle cérémonie.

La phase d'orientation est articulée autour du recrutement des facilitateurs communautaires et de l'organisation des rencontres d'orientation et d'information des facilitateurs sur les grandes

\footnotetext{
${ }^{9}$ Le mooré est la langue parlée par les mossi, ethnie majoritaire du pays mais également celle de la zone d'intervention.

${ }^{10}$ Les modules ont été développés au Sénégal et étaient rédigés en français.
} 
lignes du Programme. Des rencontres informelles ainsi que des assemblées générales de présentation et discussion des grandes lignes du programme ont eu lieu avec les autorités administratives et techniques ainsi que les responsables locaux et les représentants des villages.

La phase d'organisation a consisté à mettre en place les comités villageois de gestion et à définir le rôle et la responsabilité des membres. Avec ces comités, les critères d'identification et du choix de 30 femmes et de 30 hommes dans chaque village ont été définis et mis en œuvre.

La phase de mise en œuvre du programme Elle a consisté à former les facilitateurs sur le contenu pédagogique, à démarrer les classes pour les femmes puis les classes pour les hommes dans chaque village.

Une formation sur les règles de transcription en mooré, une formation de trois semaines sur le paquet pédagogique ont été organisées. La formation a porté sur les modules suivants : l'éducation aux droits humains, le processus de résolution des problèmes, les techniques de conduite des séances, la technique d'utilisation de la boîte à image, la pédagogie des adultes, les techniques de tenue et d'animation des séances dans les classes. Durant la seconde campagne du programme, une deuxième formation de trois semaines a été organisée. Celle-ci a porté sur les thèmes suivants: la santé de la femme, les violences faites aux femmes, l’hygiène et la prévention des maladies.

Un facilitateur a été affecté dans chacun des 23 villages et chaque village avait la responsabilité de loger son facilitateur communautaire. Chaque village a été doté de matériels et fournitures composés essentiellement de bancs, de corde et pinces à linge, de boîtes à images, de cahiers et de crayons.

Les séances d'animation ont eu lieu tous les deux jours, d'une durée de 2 heures par séance. Compte tenu du calendrier saisonnier des pluies, les 63 séances d'animation ont été dispensées aux classes des hommes et à celles des femmes au cours de deux périodes différentes.

Concomitamment aux séances dans les classes, les facilitateurs ont animé des séances publiques à l'intention des autres villageois mais également dans certains villages non couverts par le programme.

Afin de favoriser la diffusion de l'information dispensée au cours des séances, le programme d'éducation a initié le principe du parrainage qui consiste pour chaque participant à se choisir un filleul (ou une filleule) du village à qui il fait partager ses connaissances acquises.

La dernière phase est celle de diffusion de l'information.

Dans chacun des 23 villages, des réunions ont regroupé toutes les personnes et structures villageoises impliquées dans le programme ainsi que les autres membres de la communauté. Ces réunions villageoises ont également permis de préparer les forums inter-villages par aire de santé qui à leur tour ont permis aux délégations villageoises d'échanger sur les réalisations, les acquis, les forces et les limites du programme. 


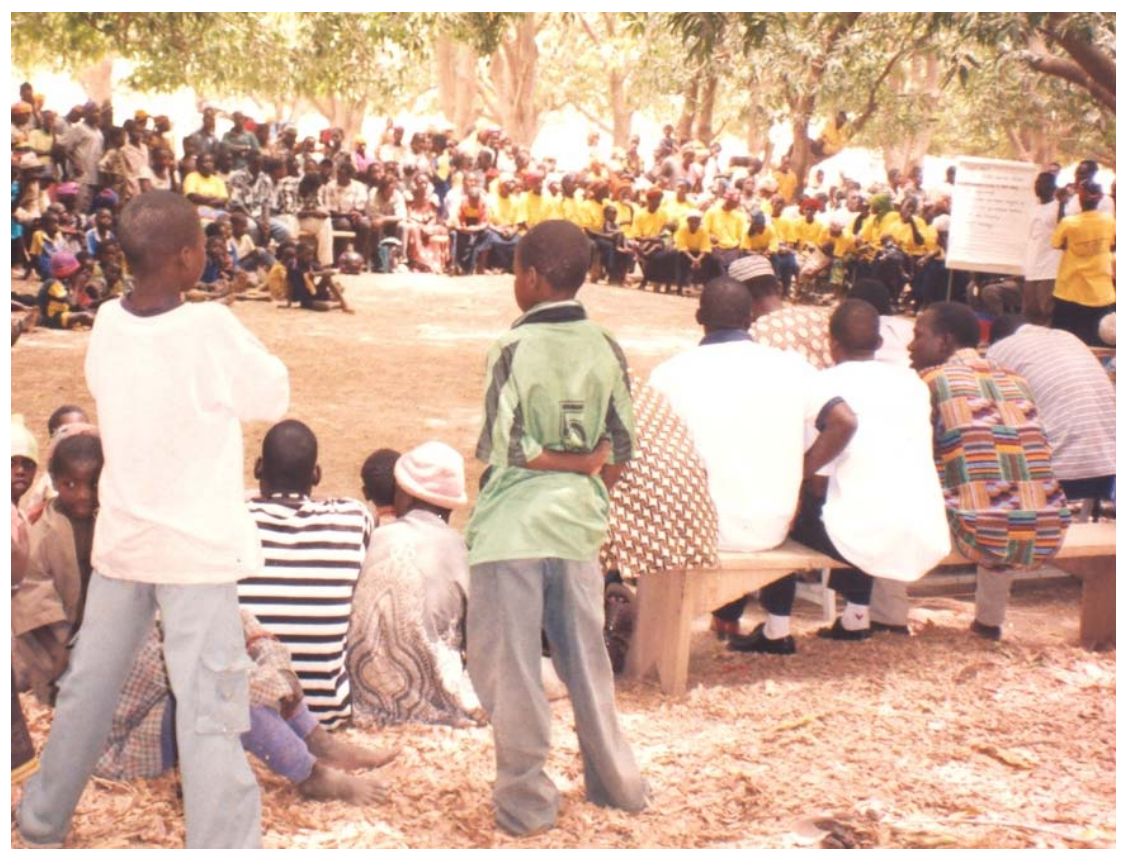

Une réunion inter-villageoise

A la fin du programme, une cérémonie de déclaration publique d'abandon de l'excision a eu lieu à Béré, chef lieu du dit département le 03 mai 2003. Cette cérémonie a regroupé les 23 villages couverts par le programme, mais aussi beaucoup d'autres personnes venues des villages environnants. On peut noter également la présence effective des chefs coutumiers et religieux de la zone. Elle a surtout été marquée par la déclaration faite par une femme au nom de toutes les communautés et le geste rituel du représentant des chefs coutumiers qui valide l'engagement pris d'abandonner la pratique de l'excision.

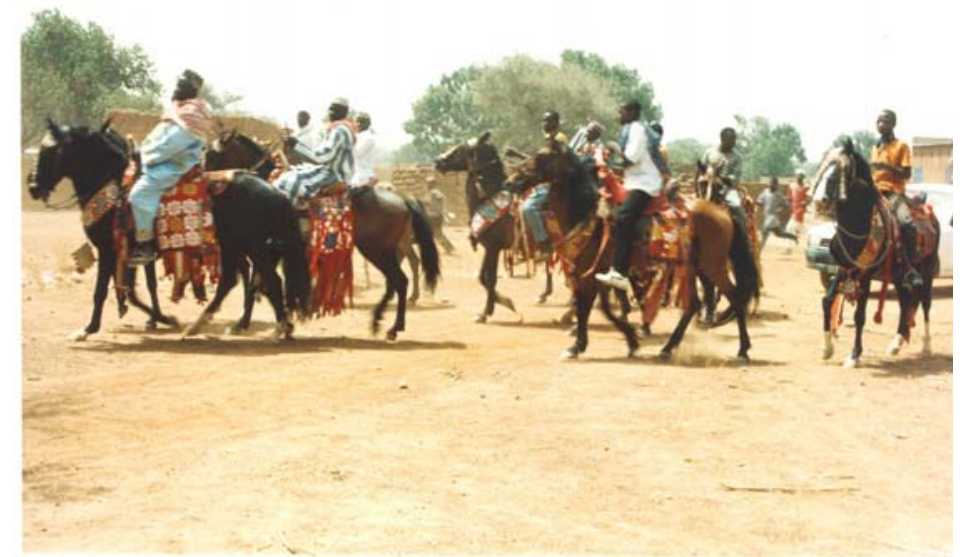

Arrivée du Moro Naba ${ }^{11}$ à la cérémonie de déclaration publique

\footnotetext{
${ }^{11}$ Chef coutumier suprême des Mossi
} 


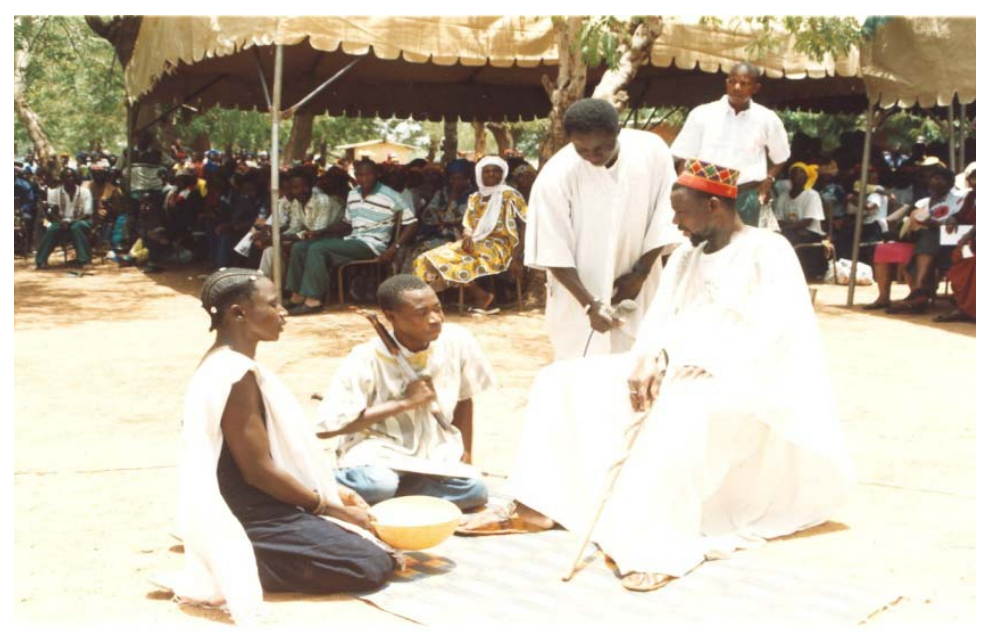

Sketch de sensibilisation sur les méfaits de l'excision

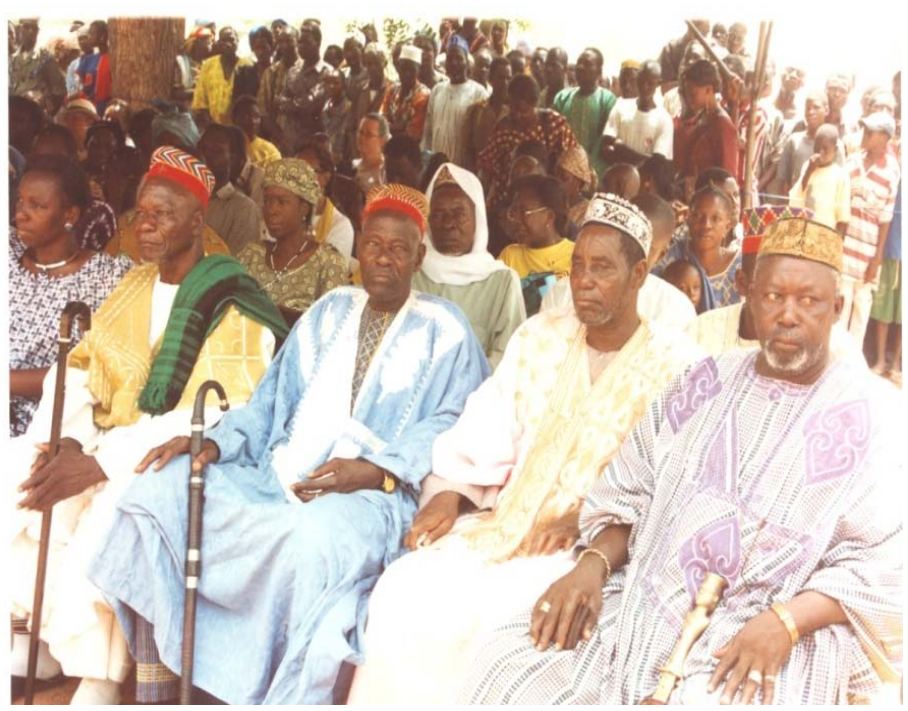

Participation des leaders religieux

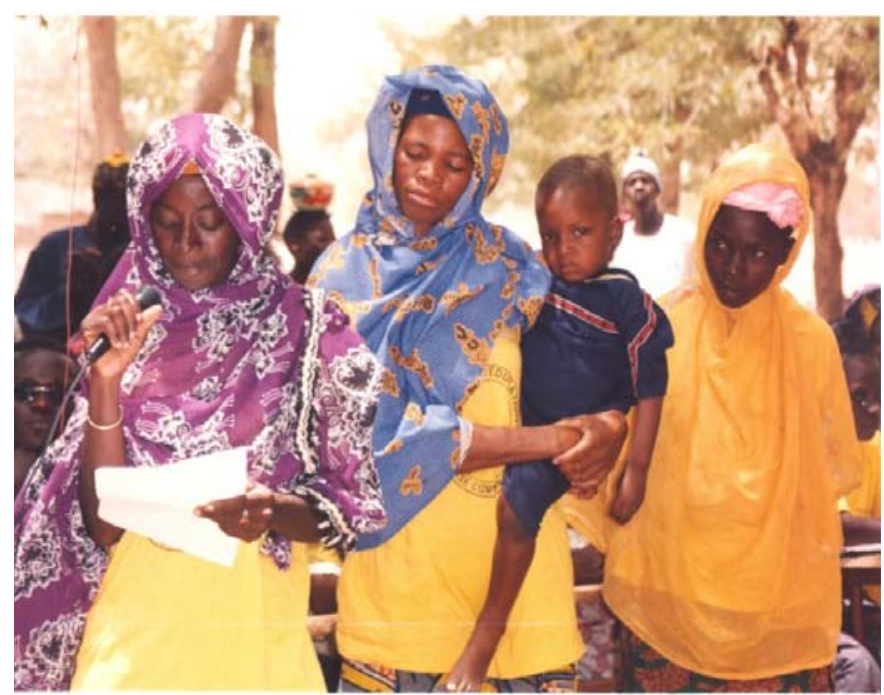

Les femmes lisent leur déclaration d'abandon de l’excision 


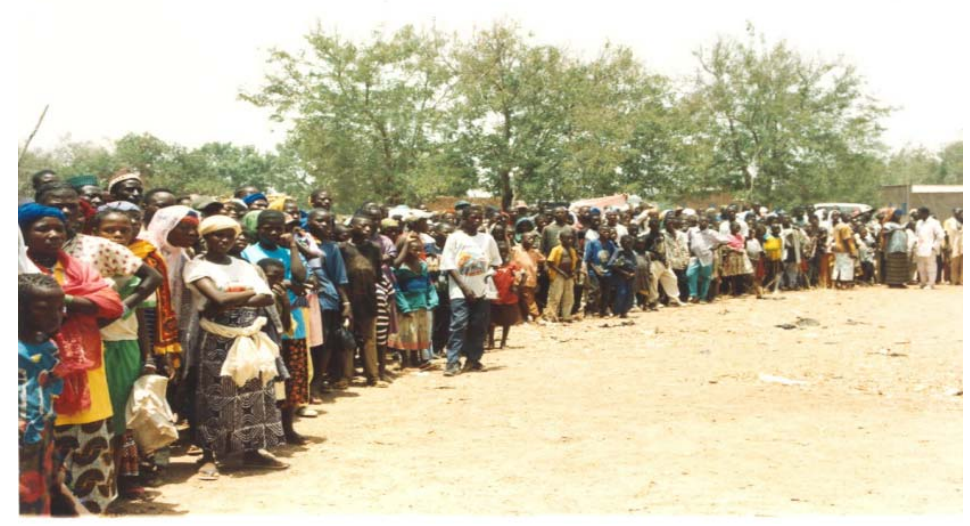

les villages environnants venus assister à la déclaration

\section{Les acquis}

\section{Au niveau communautaire}

Durant le déroulement du programme, des actions communautaires multiples ont été observées. Elles vont de la préservation de l'environnement à l'amélioration du cadre de vie, en passant par les actes civiques ainsi que les actions d'hygiène publique.

Le premier effet vise à améliorer l'hygiène du village. Les écoles, les centres de santé et de promotion sociale (CSPS), les alentours des points d'eau ont dans la plupart des villages été nettoyés. Dans l'ensemble des 23 villages, des cases de santé ont été construites pour remettre en activité les accoucheuses villageoises formées et/ou recyclées. On notera également la confection de foyers améliorés par les femmes et leurs regroupements pour mener des activités génératrices de revenu. A Béré par exemple, un nouveau comité de point d'eau fut installé et la pompe manuelle du marché, en panne depuis 3 ans, fut réparée. Certains actes civiques ont été enregistrés tels que l'établissement de jugements supplétifs d'acte de naissance, de carte d'identité et de livret de famille. Enfin, près de 300 personnes ont assisté à une cérémonie officielle où 43 couples ont scellé un mariage civil. On a pu noter également un cas de dénonciation de mariage forcé et la constatation d'un cas de discrimination par rapport à l'éducation des enfants.

Il a également été noté une prise de conscience locale des dangers de l'excision au regard des sketchs organisés par les villageois et exécutés par les femmes elles même. Et le plan d'action élaboré par les représentants des villages identifie l'excision comme une violence faite aux femmes et préconise la sensibilisation de la population sur les dangers de cette pratique.

Les actions observées ne sont pas toujours quantifiables mais elles manifestent clairement le désir des populations à s’investir davantage dans la résolution de leurs problèmes.

Cette approche donne lieu à une déclaration publique qui est l'aboutissement de la prise de conscience face au phénomène de la pratique de l'excision. 


\section{Les limites}

- La déclaration publique ne garantie pas un abandon de la pratique, notamment lorsqu'il n’y a pas un mécanisme de suivi qui permette à la société de soutenir ce processus de changement social $^{12}$;

- Le manque d'accompagnement des populations pour une poursuite des activités ;

- L'absence de mesures d'accompagnement, notamment des activités génératrices de revenus qui auraient permis aux populations de répondre à leurs préoccupations majeures signalées dans les études ;

- L'absence de système d'autopromotion permettant aux populations de continuer les activités de formation et de sensibilisation,

- La mobilité des formateurs ne permet pas de s'assurer d'une formation continue sur le temps que dure le programme.

\section{L'approche des Imams}

Cette approche a été mise en œuvre dans la province du Zoundwéogo et avait pour public cible, l'ensemble des chefs religieux. Sur le terrain, il a été constaté que les résistances se situaient au niveau des responsables religieux musulmans, c'est-à-dire les imams ; ce d'autant plus que chez la majeure partie des populations musulmanes, les motifs religieux constituent souvent l'une des principales raisons de la poursuite de la pratique.

Au bout de deux ans d'activités, il est ressorti que ceux-ci ont compris que l'excision n'était pas une obligation religieuse. Des activités ont été menées en direction de ces imams : séminaire de formation et sensibilisation individuelle.

Les imams ont mené des activités dans les mosquées, au cours des baptêmes et mariages pour informer leurs fidèles de leur erreur sur la question. Ils ont également animé une émission à la télé qui a été apprécié surtout pour le courage à le dire ouvertement. Ils ont été par la suite invités à des rencontres dans le cadre de la promotion de l'abandon de l'excision au sein du monde musulman.

\section{Les acquis}

- L'implication des chefs religieux et des chefs traditionnels dans la lutte contre la pratique ;

- L’engagement des populations cible ;

- La sensibilisation dans les mosquées ;

- La clarification de l'origine et du lien de l'excision avec la pratique religieuse ;

- La démystification de l'excision ;

- Une large diffusion des messages d'abandon de la pratique.

\section{Les limites}

- La faible motivation des leaders religieux ;

- La faible capacité de persuasion liée à la faible conviction des leaders dans la promotion des activités de lutte contre la pratique ;

- L'absence d'évaluation de cette stratégie qui ne permet pas de tirer des conclusions pertinentes sur la réussite de l’intervention.

\footnotetext{
${ }^{12} \mathrm{Cf}$. La théorie du changement social de Nahid Toubia
} 


\section{L'approche de la pair éducation}

Cette approche a été expérimentée avec une association de jeunes qui est l'APJAD (l'Association pour la Promotion de la Jeunesse Africaine et le Développement). Cette association dont le siège est à Ouagadougou travaille avec les jeunes dans la province du Kénédougou.

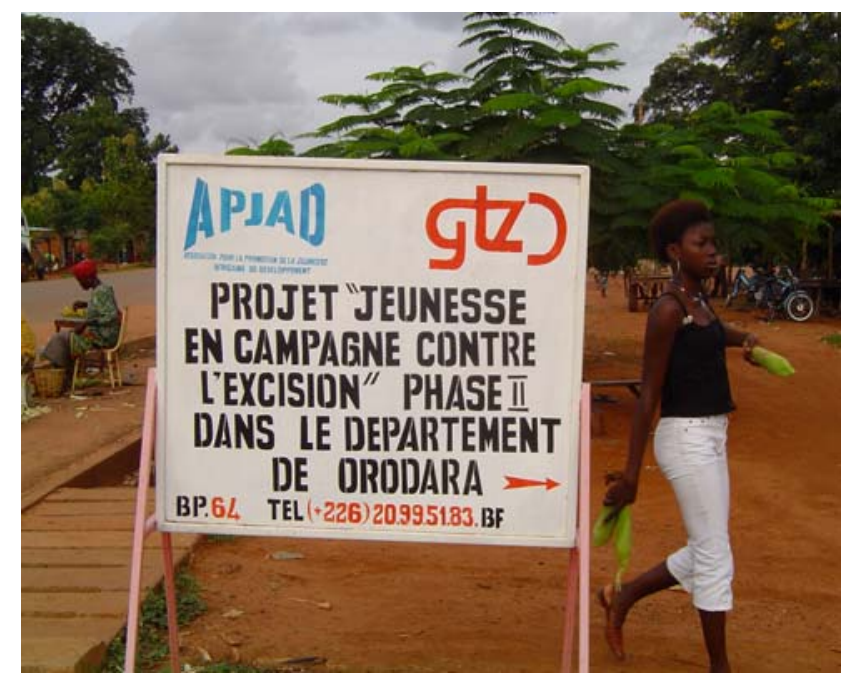

L’Association Pour la promotion de la Jeunesse Africaine et le Développement

Au niveau de l'APJAD, une étude a été entreprise dans la zone d'intervention portant sur les stratégies de mobilisation des jeunes en faveur de l'abandon de l'excision. L'étude a rassemblé des données de base sur l'étendue de l'excision et dessiné le contexte socioculturel. Les résultats ont démontré que les jeunes avaient encore clairement besoin d'informations sur l'excision. Elle a aussi donné des informations sur les réseaux et moyens de communication préférés des jeunes ce qui a servi à l'APJAD pour le planning et la mise en place de stratégies appropriées. L'étude a aussi révélé qu'il serait difficile de mobiliser les jeunes sans l'implication de leurs parents, qui jouissent d'une autorité parentale sacrée. Il a été donc décidé d’impliquer les parents et leaders d’opinion.

Le projet «Jeunesse en campagne contre l'excision» a été mis en place dans deux départements : Orodara et Korrigan. La première phase a été consacrée à mobiliser les jeunes et leurs parents tandis que la deuxième phase visait le renforcement des acquis à travers la mise en place d'un système de communication interpersonnelle permanent.

L'APJAD a choisi l'éducation de pairs comme approche principale afin d'avoir une influence positive sur les attitudes et le comportement des jeunes. Ce sont les jeunes qui offrent informations et soutien à d'autres jeunes de leur âge en matière de santé. Ces jeunes mettent en scène des pièces de théâtre pour d'autres jeunes, ils travaillent dans des centres de jeunesse et représentent les besoins de leur tranche d’âge, de sorte à mieux se sentir concernés par la campagne et encouragés à influer sur les traditions transmises et de mieux répondre au pouvoir de décision de leurs aînés. 


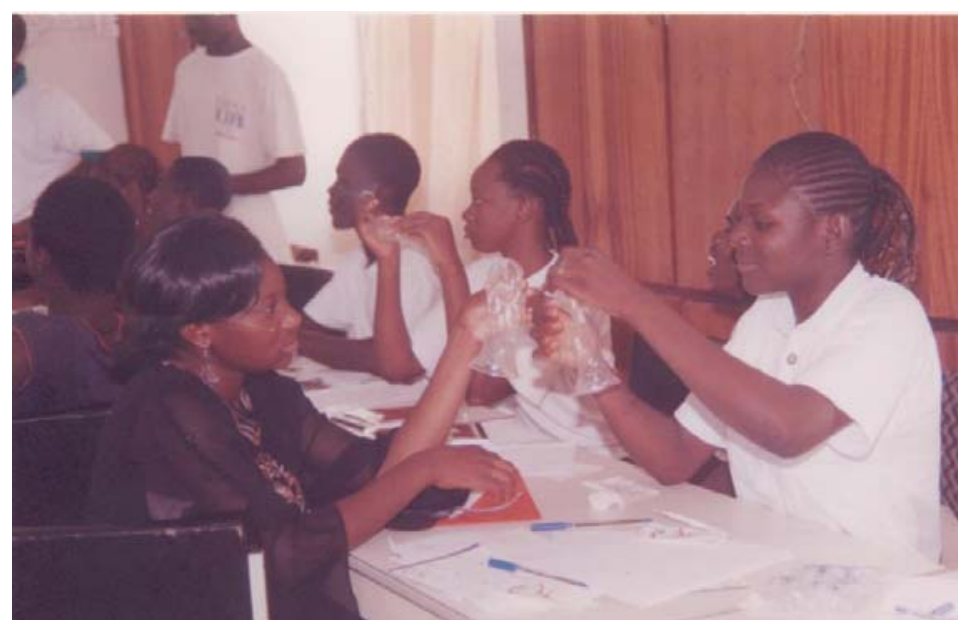

Formation des jeunes de l’APJAD en santé de la reproduction

Pour ses activités de sensibilisation, l'APJAD considère les filles et garçons de 7 à 19 ans comme public cible prioritaire, leurs parents comme cible secondaire. Mais des personnes adultes influentes telles que des leaders communautaires et des autorités administratives locales ont été mobilisés pour apporter leur soutien à la promotion de l'abandon de l'excision.

\section{La mise en ouvre}

\section{La formation des formateurs}

Au début du projet en 2000, des membres du bureau exécutif de l'APJAD ont été formés en en IEC/Excision par l'équipe de formation du CNLPE. La formation a porté sur les fondements et les conséquences néfastes de l'excision ainsi que les techniques de communication appropriées pour informer et éduquer les populations sur la nécessité d'abandonner cette pratique. Ces personnes ont eu pour tâche de former à leur tour les pairs éducateurs.

\section{La formation des pairs éducateurs}

Prévu pour former des adolescents et adolescentes de 18 à 24 ans, il a été fait recours à des jeunes adultes de plus de 24 ans pour disposer d'animateurs capables de parler en public de l'excision. Des jeunes pairs éducateurs des deux sexes ont été formés en IEC/Excision pour les rendre à même de mener des activités de sensibilisation sur le terrain à l'égard de leurs pairs.

\section{La mobilisation des personnes influentes}

Prenant en compte les réalités socioculturelles dans la zone, l'APJAD a cherché à intégrer le niveau familial et le niveau social. Ainsi, l'exécution des campagnes de sensibilisation n'a pas été uniquement confiée aux jeunes. Des encadreurs/leaders hommes et femmes ont aussi été formés pour appuyer les jeunes sur le terrain, notamment en ce qui concerne l'implication des parents. Ce groupe d'encadreurs/leaders était composé de chefs coutumiers, de délégués administratifs villageois et de leaders des groupements de femmes.

Les activités de sensibilisation

L'APJAD a utilisé une gamme de techniques de communication et de divertissements traditionnels afin d'intéresser les jeunes au thème de l'excision : pièces de théâtres forum par des groupes locaux connus autour du thème "KENEKELE TELE BANA ${ }^{13}$ ", des nuits

\footnotetext{
${ }^{13}$ Traduction : le temps de l'excision est fini
} 
culturelles (musiques et danses, messages contre l'excision avec la contribution de griots et chanteurs traditionnels), les causeries et des animations organisées les jours du marché local, projections de films suivies de discussions. Il y a eu également la distribution de T-shirts, de livre d'éducation sexuelle publié en français et en langues locales ainsi que des séances classiques de projection vidéo suivis de débats.

\section{Création et formation des clubs anti-excision}

La mise en place une stratégie de pérennisation des acquis dans les villages avec la création des équipes d'animation permanente appelées clubs anti-excision. Les activités de sensibilisation sont maintenant menées par les membres de ces clubs et le suivi est assuré par les pairs éducateurs et encadreurs.

\section{Les acquis}

Dans le village de Tin situé à $14 \mathrm{Km}$ de Orodara par exemple, le délégué administratif du village a présenté devant un public composé de femmes, d'hommes, des jeunes et des enfants, des filles non excisées baptisées "filles APJAD », car a-t-il dit, les filles nées au cours des campagnes de sensibilisation de l’APJAD ne seront plus jamais excisées.

\section{Les limites}

- Les activités menées sont limitées à deux départements, ce qui peut avoir un impact limité dans la zone, d'autant plus que la province est reconnue comme étant un bastion de la pratique de l'excision ;

- Le manque d'évaluation de la stratégie ne permet pas de tirer des conclusions fiables quant à l'impact réel des activités dans les zones couvertes, d'autant plus que l'excision s’y pratique à un âge beaucoup plus élevé.

\section{L'approche des leaders communautaires}

\section{Contenu}

L'étude de base sur la pratique de l'excision dans cinq provinces du Burkina Faso menée en 2000, a identifié le Yatenga dans la région du Nord comme la province la plus touchée par la pratique de l'excision dans tout le Burkina Faso. L'analyse approfondie de la situation a montré que la pratique de l'excision persiste dans la province du Yatenga à cause de la pratique religieuse, de forte pression sociale, de situation frontalière avec le Mali et d’insuffisance de connaissance des droits humains et des conséquences de l'excision.

Le Projet a donc apporté un appui à l'association A.M.M.I.E (Appui Moral, Matériel et Intellectuel à l'Enfant) qui a été retenue comme une des structures partenaires à cause de son approche de renforcement des activités de promotion de l'abandon de la pratique de l'excision à travers les leaders communautaires, hommes et femmes.

Conception de la pratique/ Approche utilisée

Pour aboutir à un changement des comportements de la part des leaders traditionnels ayant forte tendance à maintenir la pratique ancestrale, A.M.M.I.E. a choisi d'adresser et d'impliquer davantage les différents leaders communautaires: les femmes responsables d'associations et des groupements, les chefs coutumiers et religieux, les responsables administratifs, les ex-exciseuses entre autres. Ces leaders communautaires ont été formés comme animateurs/trices relais pour mener des activités de sensibilisation et d'éducation 
contre la pratique de l'excision dans leurs familles et communautés. Le but essentiel de cette approche est de garantir une appropriation et une prise en charge des activités d'animation et de sensibilisation par les populations à la base. Compte tenu de sa zone de couverture l'association a retenu cinq départements (Kossouka, Ouahigouya, Oula, Séguénéga, Thiou) et trois communes (Ouahigouya, Séguénéga, Thiou).

\section{L'organisation de concertations provinciales et départementales}

Pour toucher dès le départ un grand nombre de personnes influentes, l'association a choisi de démarrer les interventions du projet par des concertations provinciales réunissant les décideurs de la province (responsables politiques, administratifs et de médias, coutumiers, religieux et des services de l'ordre, des représentants des jeunes, des femmes, d'associations de développement et de défense des droits de l'homme). La rencontre avait pour but d'amener les participants à prendre conscience des méfaits de la pratique de l'excision et à analyser les fondements de la pratique et à amener les participants à déterminer les actions à entreprendre, les stratégies à adopter, les acteurs indiqués, les messages appropriés, le public cible etc.

\section{L'organisation de journées d'agitation}

Des journées d'agitation ont été organisées, en réponse à la journée de marche des femmes de Ouahigouya de fin 2000 qui avait été organisée contre la lutte engagée et pour demander la libération d'exciseuses et de parents d'enfants excisés arrêtés. Elle a été une occasion de lancer un débat public sur la question de la légitimité de la pratique.

\section{Le renforcement des capacités}

Des formations et des recyclages ont été organisées pour les présidentes des groupements féminins, les personnes ressources des départements (responsables coutumiers et religieux), les leaders communautaires (préfets, agents de santé, responsables de jeunes, de femmes, directeurs de lycées, représentant des forces de l'ordre, membres des comités villageois, exciseuses) et les membres de l'association, notamment les femmes.

\section{Les activités de sensibilisation}

Des activités de sensibilisation ont été menées à travers des causeries débats, l'animation de débats de sensibilisation au niveau des radios locales (avec possibilités d'intervention directes des populations pour poser des questions, présenter et discuter leur position, leurs croyances et leurs craintes) que sont «Voix du Paysan », « Notre Dame du Sahel » et "Radio de l'Amitié », toutes basées à Ouahigouya. Il faut noter également les activités telles que l'organisation de concours de chants et de pièces théâtrales avec les troupes locales, l'organisation de spectacles d'animation dans les départements, les projections de films.

En plus de ces activités, la stratégie de la sensibilisation de proximité a été mise en place avec notamment le porte à porte, entretien individuel ou par famille, la mise en place de relais communautaires et comités villageois qui assument le rôle de comité de surveillance et de vigilance afin de détecter à temps les cas d'excision en préparation et le recensement et la sensibilisation des exciseuses.

\section{La journée de déclaration publique d'abandon de l'excision}

En juillet 2003, des délégués de tous les villages couverts par le projet se sont déplacés à Ouahigouya pour faire une déclaration collective et publique d'abandon de la pratique. Cette journée a connu la participation des populations, des autorités administratives locales, des autorités coutumières et religieuses ainsi que les partenaires du projet. 


\section{Les acquis}

- L'engagement de premier ordre de la part de la responsable de l'association qui est à la fois député et membre du parlement de la CEDEAO ;

- Le renforcement des compétences des leaders communautaires (les responsables des groupements féminins, les personnes ressources, les chefs coutumiers et religieux) des villages d'intervention et des animateurs et animatrices de l'association ;

- L'implication effective de ces leaders communautaires dans la lutte contre la pratique de l'excision, signe d'un fort engagement communautaire, gage d'une appropriation de la lutte contre l'excision par les populations à la base ;

- La mise en place de relais communautaires et de comité villageois de vigilance et de surveillance pour dénoncer les intentions d'excision ;

- La participation des leaders femmes aux activités de sensibilisation, donnant une occasion d'amélioration de leur statut social ;

- L'intégration de la lutte contre l'excision dans d'autres activités de développement de l'association (poste de santé primaire, activités de santé maternelle et infantile, alphabétisation, etc) ;

- La sensibilisation continue avec les chansons et pièces de théâtre comportant des messages sur les conséquences de la pratique de l'excision ;

- La déclaration publique d'abandon de la pratique de la part des délégués des villages de la zone d'intervention.

\section{Les limites}

- L'absence d'évaluation des activités de cette stratégie, notamment sur la fonctionnalité des relais et comités villageois de surveillance.

- L'absence d'évaluation après déclaration publique pour mesurer le changement de comportement.

\section{L'approche de l'intégration des modules dans le cursus scolaire}

La responsabilité est à la Direction de l'Education en Matière de Population (DEMP). Elle est une des Directions Centrales du Ministère des Enseignements Secondaire, Supérieur et de la Recherche Scientifique chargée d'introduire les questions démographiques, la vie familiale et la sexualité dans les programmes scolaires et universitaires.

En termes de contenus, les cours traitant de sujets liés à la population et la santé reproductive prennent en considération les questions de santé de l'individu, spécialement celle des femmes et des filles, les aspects éthiques, le contexte socioculturel et les pratiques traditionnelles.

Pour parvenir à l'objectif général qui est l'intégration de la lutte contre l'excision dans les programmes d'éducation scolaire primaire et secondaire, un processus d'expérimentation a été planifié dans 12 écoles pilotes (6 du primaire et 6 du secondaire) situées en zone urbaine et rurale de 6 Directions Régionales de l'Education (DRE). Les provinces et leurs chefs-lieux retenus ont une certaine représentativité par rapport aux spécificités socioculturelles du pays : Kadiogo (Ouagadougou) dans le centre ; Gourma (Fada-N'Gourma) à l'Est ; Boulkiémdé (Koudougou), dans le centre-ouest ; Houet (Bobo-Dioulasso), Comoé (Banfora) dans l'ouest et Poni (Gaoua) dans le sud-ouest.

Le groupe cible principal de l'approche est constitué des élèves du primaire et du secondaire. D’autres groupes cible sont les enseignant(e)s, les encadreurs pédagogiques et les inspecteurs 
des écoles pilotes ainsi que les parents d'élèves. L'intégration du thème de l'excision à travers des formations utilisant des matériels et supports appropriés vise à marquer durablement les membres de toute la communauté éducative et surtout les élèves pour un abandon de la pratique de l'excision.

\section{La mise en oeuvre}

\section{La réalisation d'une étude de base}

Les activités ont été lancées en octobre 2000 avec une étude destinée à collecter des données de base dans des écoles primaires et secondaires sélectionnées dans la région du projet. L'étude devait mesurer les connaissances, attitudes et comportements des élèves et enseignants ainsi que ceux des parents d'élèves relatifs à l'excision.

\section{L'élaboration de modules, fiches pédagogiques et autres matériels didactiques}

Un module de formation destiné aux enseignants du primaire et un autre destiné à ceux du secondaire ont été élaborés en novembre 2000 par une équipe composée de conseillers pédagogiques et d'instituteurs certifiés pour le niveau primaire et une équipe d'enseignants de plusieurs disciplines pour le secondaire pour leur intégration dans les disciplines suivantes : français - histoire - géographie - sciences naturelles - économie sociale et familiale, philosophie. Le matériel didactique comprend un recueil de notes de lecture, des fiches pédagogiques, des dépliants, des diapositives, des planches, des cassettes vidéo ainsi que des affiches, etc. le développement des modules est basé sur l'application des Méthodes de Résolution des Problèmes et de la Méthode de Clarification des Valeurs qui développent l'esprit critique et la prise de conscience des élèves. "

Les supports didactiques amendés ont été multipliés et mis à la disposition des enseignants expérimentateurs et de leurs encadreurs. Le CNLPE a soutenu les expérimentateurs en mettant à leur disposition du matériel de sensibilisation (planches, mannequins, dépliants, tee-shirts, guides, cassettes...).

\section{L'implication des parents d'élèves}

Une fois ces matériels achevés, une campagne de sensibilisation a été lancée en direction des parents d'élèves des écoles pilotes. Il s'agissait de les informer sur le contenu des nouveaux cours qui seront dispensés sur l'excision et de les impliquer parfois lorsqu'il faut dispenser le cours pour discuter des valeurs culturelles liées à la pratique de l'excision.

L'organisation d'un atelier de formation des enseignants expérimentateurs

Pour préparer les enseignants à leur tâche dans les écoles pilotes, un séminaire atelier de formation des enseignants expérimentateurs des établissements pilotes a été réalisé en Juillet 2001. Il s'est agit de les former à l'utilisation des modules et matériels didactiques relatifs au thème ainsi qu'aux instruments d'évaluation des attitudes.

\section{L'expérimentation proprement dite}

Les modules, recueils de fiches pédagogiques et autres matériels didactiques ont été intégrés dans l'enseignement des 12 écoles pilotes pour la phase d'expérimentation pendant une année scolaire de septembre 2001 à Juin 2002. Des visites de suivi ont permis de suivre les prestations des enseignants. 


\section{Les acquis}

- Les élèves dans leur grande majorité sont plus informés sur les questions relatives à l'excision ;

- Une facilité d'aborder les questions liées à l'excision chez les parents d'élèves, notamment au sein de leur association et davantage avec leurs enfants une fois à la maison ;

- La signature d'une lettre circulaire conjointe des deux ministères chargés de l'enseignement primaire et secondaire, portant sur la prise en compte des modules élaborés sur l'excision dans les programmes scolaires et leur intégration dans les établissements primaires et secondaires du pays ;

- La disponibilité des compétences aussi bien au niveau central que décentralisé ;

- Un engagement politique réel avec la prochaine intégration du programme dans le plan de développement de l'éducation de base (PDDEB).

\section{Les limites}

- L'insuffisance d'équipement des écoles primaires en zone rurale où l'électricité n'est pas disponible pour la projection des films et diapositives ;

- La mobilité des enseignants pour cause d'affectation dans des localités hors zone expérimentale qui nécessite le remplacement des enseignants déjà formés ;

- La limitation actuelle du projet au niveau des écoles pilotes ;

- L'absence de ces modules dans le programme de formation des instituteurs et des enseignants du secondaire ce qui aurait eu pour effet d'avoir des enseignants ayant les capacités d'intégration de la question de l'excision avant leur sortie sur le terrain.

\section{Le Projet Mille Jeunes Filles}

Un programme novateur à l'intention des adolescentes rurales intitulé Projet des centres de production et de formation pour jeunes filles $(\mathrm{CPF} / \mathrm{JF})$ a été mis en œuvre dans le Sud-ouest. Il est communément appelé projet 1000 jeunes filles ${ }^{14}$. Ce projet est issu de la mise en oeuvre des engagements pris en 1994 par le chef de l'Etat dans le cadre de l'amélioration des conditions de vie des femmes et de la population en général. Aussi le projet reste sous le couvert des Engagements Nationaux mais relève de la tutelle technique du Ministère de l'Action Sociale et de la Solidarité Nationale.

Il vise particulièrement des adolescentes de 14 à 18 ans. Ces jeunes filles sélectionnées à travers l'ensemble du pays sont regroupées dans deux centres de formation et de production (Niassan et Debé) durant deux ans. Elles y apprennent un métier et on leur donne des connaissances dans d'autres domaines comme la santé de la reproduction, l'excision, l'hygiène et l'assainissement, l'alphabétisation. L'objectif ultime de projet est de contribuer à améliorer les conditions socio-économiques de la jeune fille et des femmes en général. Après avoir passé deux ans au niveau des centres de production, les filles rejoignent leurs familles respectives.

\footnotetext{
${ }^{14}$ Le centre est supposé accueillir 1000 jeunes filles pour la formation, d’où cette appellation.
} 


\section{La mise en ouvre}

Le démarrage du projet a été fait de manière rapide. Les structures décentralisées de l'Action Sociale et les autorités administratives (Haut commissaire et préfet) ont été mises en contribution pour un recrutement rapide des filles à former. Chaque département avait pu identifier trois filles de 14 à 18 ans. Approximativement mille filles ont été recrutées.

Les filles sélectionnées ont été regroupées au niveau de la province du Sourou. Elles ont été réparties dans les deux centres suivant l'âge. Toutes les filles ont suivi par rotation toutes les matières enseignées durant la toute première année : teinture, couture, tissage, maraîchage et éducation à la vie familiale y compris l'excision. Chaque fille choisit par la suite une matière de spécialisation pour la dernière année. L’exécution des activités a connu la collaboration de plusieurs ministères et celle de quelques institutions internationales telles que l'OMS, l’UNFPA, la Coopération Chinoise.
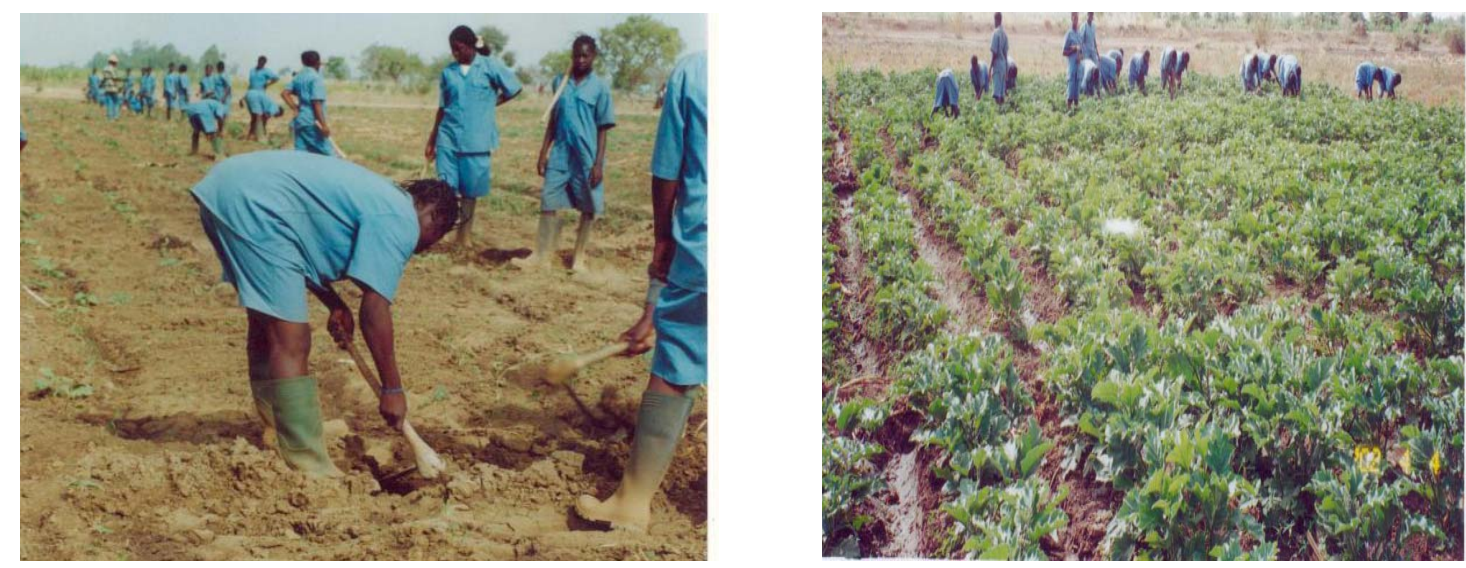

Les filles du projet mille jeunes filles apprenant le maraîchage

Outre l'attestation de fin de formation, chaque fille reçoit une dotation en équipement correspondant à son domaine de spécialisation et un carnet d'épargne avec la somme de 120 000 FCFA. Cette somme correspond en faite à une épargne de 10 000FCFA effectuée durant les deux ans sur le pécule mensuel qui était alloué à chaque fille.

Dans la perspective de l'intégration des filles au niveau de leur communauté, un comité provincial de suivi placé sous la présidence du Haut commissaire a été mis en place afin de permettre aux filles d'avoir facilement accès aux conditions leur permettant d'exercer une activité, en l'occurrence celle apprise au centre. Dans le même sens, une marraine a été identifiée dans chaque province pour assurer le suivi et constituer en même temps un chaperon pour les filles de la province.

\section{Les acquis}

- Le suivi de la grossesse, la vaccination des enfants constituent les grands résultats de cette formation.

- Aucune fille sortie du centre de formation n'avait pratiqué l'excision de leur enfant et la plupart des filles affirment ne pas avoir excisé leur petite fille à cause des enseignements appris sur l'excision dans les centres de formation. 


\section{Les limites}

- Le manque de suivi réel des filles sorties du centre

- Le faible pouvoir de décision au sein de la famille (dépendance économique et sociale)

- L'excision n’apparaît pas comme un élément fondamental dans les questions de Santé Reproductive abordées pendant la formation.

\section{Le renforcement des capacités des adolescentes}

Mis en oeuvre par le ministère de la santé et appuyé par le Population Council, le projet UNF est une expérience pilote au Burkina Faso. Il s’intitule «Renforcement des services sociaux et sanitaires pour satisfaire les besoins en Santé de la Reproduction des adolescentes ». Le projet intervient principalement en milieu rural, dans les districts sanitaires de Kombissiri et de Fada ; notamment à Kombissiri, Gaongo et Naaf-banka (dans la province du Bazèga), à Fada, Natiaboani et Koaré (dans la province du Gourma).

Le projet vise à renforcer les " compétences de vie » et les droits au développement et à la santé, à rendre disponibles et accessibles les services de santé sexuelle et reproductive et à améliorer la qualité de vie de ces adolescentes (mariées ou non mariées) âgées de 10 à 19 ans. La question de l'excision y occupe une bonne place.

\section{La mise en ouvre}

Elle s'articule autour de cinq aspects i) la création d'un environnement social et politique favorable à la mise en œuvre du projet par le plaidoyer auprès des leaders d'opinion, des dirigeants communautaires, le développement d'un réseau d'ONG/associations. ii) l'IEC à travers la formation des paires éducatrices, la communication avec la communauté, l'utilisation des canaux de communication tels les représentations théâtrales, iii) l'offre des services socio-sanitaires de qualité accessibles en SR/SS cela passe par la formation des prestataires de service, iv) l'amélioration de l'accès aux produits contraceptifs y compris par l'approche de la DBC, v) le développement d'une base de données pour la gestion, le suivi et l'évaluation du projet il s'agit de mener une analyse de la situation pré-intervention , une étude CAP et une revue des données sur les caractéristiques des groupes cibles, des politiques et programmes.

Les approches privilégiées qui sous-tendent ces stratégies sont i) la participation de la communauté à travers un plaidoyer en vue d'asseoir une base solide au développement des autres aspects, ii)l'utilisation des paires éducatrices mariées et non mariées permettant de transmettre les messages sur la SR et SS iii) le renforcement des espaces socio-sanitaires existant visant à augmenter le taux de fréquentation par les adolescents et les jeunes au niveau des villages (Saloucou et al., 2002) .

Après la première phase, le projet a changé de dénomination et est devenu le projet PSADO. Celui-ci s'appuie essentiellement sur les acquis du projet pilote à savoir (i) un faisceau de trente pairs éducatrices et de membres d'associations, formés et équipés qui animent et sensibilisent sur des thèmes de SRAJ, dans toute la zone d'intervention ; (ii) un réseau DBC opérationnel dans toute la zone d'intervention qui cible prioritairement les adolescentes et les personnes clés dans leur vie ; (iii) la conduite réussie de douze centres d’alphabétisation de niveau 1; (iv) des agents/personnels formés et des services renforcés pour répondre aux attentes des adolescents ; (v) et surtout, une large mobilisation sociale en faveur de la SRAJ impliquant les leaders communautaires (femmes et hommes). 
Les stratégies du projet consistent en :

- La création d’un environnement social favorable au projet ;

- Le renforcement des capacités et des compétences de vie, y compris en matière de santé sexuelle et en santé de la reproduction ;

- Le renforcement de l'accessibilité à des services sociaux et sanitaires de qualité, en particulier des services de SR ;

- Le renforcement et la pérennisation de l'accès des adolescentes et des jeunes aux produits contraceptifs ;

- Le transfert des leçons apprises vers les autres projets jeunes.

\section{Les acquis}

- Une importante mobilisation communautaire et une adhésion de toutes les couches sociales aux objectifs du projet ;

- Des services sociaux et sanitaires renforcés, en compétence et en matériel, et réhabilités pour assurer des services de qualité ;

- Une disponibilité de services à base communautaire délivrés par trente pairs éducatrices ;

- Une importante base de données sur les adolescentes mettant en évidence :

- La précocité du mariage, des rapports sexuels et de la grossesse, avec une proportion importante des accouchements qui se font sans assistance qualifiée ;

- L'absence d'information des adolescentes entrant dans le mariage sur les méthodes de prévention des IST/VIH/SIDA, la grossesse et l'excision ( 7,6\% des adolescentes et 19,3\% des adolescents déclarant avoir excisé ou compte exciser leur fillette).

- Une méconnaissance des signes de danger au cours de la grossesse et de l'accouchement au niveau des communautés ;

- La limite des capacités/qualifications pour influencer les décisions dans leurs propres vies ;

- Le manque d'autonomie financière et d'opportunité de développement économique pour assurer les coûts liés à l'accès aux services de santé pour renforcer leur statut dans le ménage et la société, etc.

\section{Les limites}

- Les activités relatives à l'excision sont noyées dans un ensemble d'activités.

- Le manque de conviction des relais communautaires qui ne mettent pas beaucoup l'accent sur l'excision.

\section{Conclusion}

La mise en place d'un environnement favorable à la lutte contre l'excision a donné l'occasion pour beaucoup d'interventions sur le terrain. En effet, plusieurs études ont été réalisées, ce qui a permis d'avoir des actions coordonnées et d'agir de manière efficace. Les différentes conventions signées ont également donné un caractère international aux actions et activités de promotion de l'abandon de la pratique. L'adoption de la loi contre la pratique de l'excision et son application ont été déterminant dans la lutte. A cela s'ajoute la grande volonté politique de l'état qui n’a ménagé aucun effort pour soutenir la lutte avec notamment la prise en charge 
du téléphone vert. De plus, la mise sur pied du CNLPE impliquant tous les ministères a permis d'avoir une vision holistique, ce qui accroît l'efficacité des interventions.

Les interventions au Burkina Faso sont diverses et touches plusieurs publics. Il s'agit aussi bien des jeunes, des adultes, des leaders communautaires, religieux, et politiques, les forces de sécurité, les forces judiciaires. Cette implication de toutes les catégories de la société a accrue l'efficacité des activités de lutte contre la pratique contre l'efficacité. Il y a un grand effort fait par le gouvernement pour infiltrer tous les corps de métiers de la société et toutes les personnes influentes. Ces actions ont permis d'atteindre beaucoup d'acquis aussi bien en termes de connaissance mais également en termes de changement de comportement.

L'utilisation des médias est constante dans le processus. Leurs actions continues ont fortement participé à la sensibilisation et à la conscientisation des populations sur les méfaits de l'excision.

Cependant, force est de constater que la majeure partie des activités et actions des différentes interventions sont surtout axées sur la sensibilisation de masse. Quelques stratégies de proximités sont développées sans pour autant être véritablement centré là-dessus. En dehors des actions en direction des jeunes qui représentent une cible particulière, les autres actions concernent le grand public. A ce niveau, il faut davantage spécifier les stratégies pour chaque cible pour améliorer la qualité des résultats. Ce passage d'un discours trop général à un autre plus spécifique est un impératif et devrait permettre d'optimiser les résultas des actions menées. 


\section{Références bibliographiques}

Centre pour le droit et les politiques en matière de santé et de reproduction (CRLP). 2000. Les droits de la personne: un outil pour combattre les mutilations génitales féminines, Guide pratique au service des militants avril, 60 pages

Comité National de Lutte contre la Pratique de l'Excision (CNLPE). (1999). Plan d'action de lutte contre la pratique de l'excision (1999-2003),Ouaga, 39 pages + Annexes.

Comité National de Lutte contre la Pratique de l'Excision (CNLPE) 2003. Bilan des actions du CNLPE 1990-2003, mai, 23 pages

Comité National de Lutte contre la Pratique de l'Excision (CNLPE). 2004. Rapport narratif des patrouilles de sensibilisation et de dissuasion réalisées par vingt deux (22) brigades de gendarmerie, Octobre, 10 pages

Comité National de Lutte contre la Pratique de l'Excision (CNLPE). 2005 : Rapport de la rencontre de concertation des acteurs de la mise en œuvre des activités financées par l’Ambassade Royale des Pays -Bas

Kaboré Gisèle, Sobela Suzanne, Saloucou Lydia. 2002. Revue des politiques et des programmes sur la santé de la reproduction des adolescents au Burkina faso, UNFPAPopulation Council, Ouagadougou, 98 pages.

Kessler Bodiang, Claudia. 2003. La lutte contre les mutilations génitales féminines, défis et perspectives pour les programmes de santé; première partie: une sélection d'approches, GTZ, février, 42 pages.

Konaté Alimata, 1998. Rapport de l'étude qualitative sur les mutilations génitales féminines dans douze (12) villages Plan du Sud-Ouest, Ouagadougou, Mai, non paginé

Organisation Mondiale de la santé. 1996. Mutilations sexuelles féminines : Rapport d'un groupe de travail technique de l'OMS, Genève 17-19 juillet 1995, Genève, 30 pages

Ouédraogo Amélie, 1998. Etude qualitative sur la pratique des mutilations génitales féminines dans dix (10) villages Plan de chacune des provinces du Bam et du Sanmatenga, Ouagadougou, juin, 48 pages + annexes

Ouédraogo M.Louise et Tapsoba Lydia. 2005. Evaluation interne du projet lutte contre la pratique de la mutilation génitale féminine dans les régions de l'Est et du Nord du Burkina Faso, février, Médicus Mundi Andalucia, non paginé.

Sebgo Pascaline. 1999. Evaluation des initiatives contre les mutilations génitales féminines et proposition de la composante du projet supra régional au Burkina Faso, Rapport de mission, Ouagadougou, 108 pages

UNICEF. 2003. Activités du plan intégré de communication sur les mutilations génitales féminines et le code des personnes et de la famille, rapport synthèse, Ouagadougou, juin, 9 pages

Toubia Nahid, Rahman Anika. (2000). Female Genital Mutilation: A Guide to Laws and Policies Worlwide, New York, Rainbo, 249 pages. 